\title{
Frank Mußmann \\ Die Mindesteinkommensdiskussion, Überwindung der kapitalistischen Rationalität durch Entkopplung von Arbeit und Einkommen?
}

„Die Verteilungsmechanismen müssen ganz neu überdacht werden. Die gewaltige Wertschöpfung in den Fabriken muß gerecht auf die Menschen verteilt werden. Es ist fraglich, ob der Maßstab des betrieblichen Arbeitslohnes hierfür noch zureichend ist.\&

Albrecht 1983

„Was ist $z$ tun, wenn der Produktionsprozeß immer weniger Arbeit erfordert und immer weniger Löbne ausschüttet?... Das Recht auf Einkommen muß einem jeden zugestanden werden, und die Kaufkraft des einzelnen darf nicht mehr von der erbrachten Arbeitsmenge abbängen. Das Recht auf Einkommen kann also nicht länger vom Besitz eines Arbeitsplatzes abhängig gemacht werden."

Gorz 1986

Was ist passiert? - Sind dies die Vorboten eines redlichen Klassenkompromisses jenseits des Kapitalismus, mithin einer 'postkapitalistischen Gesellschaft', die diesen Namen verdiente oder hat der Diskurs der Herrschenden eine qualitativ neue Stufe der 'Naturalisierung von Geschichte durch Mythologisierung' erreicht, indem er sich der Sprache der Beherrschten immer mehr annähert und deren Sinn 'durch Diebstahl kolonialisiert' (vgl. Barthes 1982; zum 'Themenraub' vgl. Wiesenthal 1985)? Wenn auch das zweite wahrscheinlicher sein dürfte als das erste, so ist es in diesem Fall mit einer ideologiekritischen Argumentation allein sicher nicht getan. Es sind reale Veränderungen des gesellschaftlichen Reproduktionsmodus, die den Protagonisten beider antagonistischer Lager die Basis für ihre auf den ersten Blick ähnlichen Überlegungen liefern.

Spätestens seit dem gleichzeitigen Nebeneinander von positiven Wachstumsraten der gesellschaftlichen Produktion und den steigenden Arbeitslosenzahlen verweist die gesellschaftliche Realität in den 80er Jahren auf eine 'Krise der (Erwerbs-)Arbeitsgesellschaft', quer durch alle Ideologien, zumindest aber verweist sie auf eine Krise des Beschäftigungssystems. Der Funktionsverlust des Arbeitsmarktes bei der Verteilung von Arbeitsplätzen (Systemintegration) und der auf Einkommen basierenden Lebenschancen (Sozialintegration) setzt in der Tendenz auch die letzten materiellen Bezugspunkte der Leistungsideologie außer Kraft. Trotz aller ideologischen Kraftanstrengungen droht die (Lohn-)Erwerbsexistenz ihre Bedeutung als 'Normalexistenz' zu verlieren und kann darum nicht länger als Regelfall der sozialpolitischen Administration verstanden werden: In einer Zeit, in der dauerhaft weit mehr als ein Zehntel der abhängigen Erwerbspersonen aus dem Beschäftigungssystem und darüber hinaus zunehmend auch aus der Arbeitslosenversicherung ausgegrenzt werden, belegt der dadurch sich notwendig einstellende Prozeß der Verarmung durch Arbeitslosigkeit besonders drastisch die Überkommenheit einer lohnarbeitszentrierten Sozialpolitik.

Die Realität des bestehenden Sozialsystems tritt dabei in zweifacher Weise zunehmend in einen unvermittelten Widerspruch zu seinen inhärenten Prinzipien, die, durchgängig orientiert an den Belangen der kapitalistischen Arbeitsgesellschaft, u.a. in sozialrechtlichen Bestimmungen institutionalisiert sind: 
- Die arbeitszentrierten Vorbehalte des sozialen Sicherungssystems werden immer sinnloser, wenn die Reduktion der Erwerbsarbeit immer mehr Menschen die Chance verstellt, jemals in abgesicherter Form an der gesellschaftlichen Produktion teilzunehmen. Die vielfältigen Prinzipien des Arbeitszwangs, die allesamt darauf ausgerichtet sind, Existenzmöglichkeiten ausschließlich im Medium von Lohnarbeit (bzw. der erklärten Bereitschaft dazu) zu sanktionieren, werden absurd, wenn gesellschaftlich die Probleme des Abbaus der lebendigen Arbeit zu bewältigen sind. Ohne Relativierung der Normen der (Erwerbs-)Arbeitsgesellschaft könnte gerade diese Tradierung der notwendigen Adaption entgegenstehen.

- Zum anderen wird die bestehende Form der sozialen Sicherung zunehmend ineffektiv, weil es gerade die Funktionsprinzipien des Arbeismarktes und die spezifische Selektivität der sozialrechtlichen Leistungsgewährung sind, die wechselseitig das Entstehen von Armut bedingen (vgl. Hanesch 1984, S. 122 f.).

Mit Regelmäßigkeit stellt sich in Krisenzeiten, jeweils angekündigt durch eine 'Finanzkrise', alsbald auch die 'Krise der Sozialpolitik' ein: Zum einen führen sinkende Lohnsummen zum Rückgang der Einnahmen von Staat und Sozialversicherung, zum anderen führt das Anwachsen der Anzahl der aus dem Erwerbsleben Ausgegrenzten zur Steigerung der Leistungsanforderungen an das soziale Sicherungssystem. Dessen innere Logik (A bhängigkeit der Sekundär-von der Primärverteilung) führt bei immer mehr Anspruchsberechtigten zu chronischer Unterversorgung: Entweder werden dem Versicherungs- und Äquivalenzprinzip folgend Unterhaltszahlungen an das letzte Arbeitseinkommen ohne Berücksichtigung jeglicher Bedarfsgesichtspunkte gekoppelt, oder - wie in der Sozialhilfe - einem nur als Rudiment erhaltenen Bedarfsprinzip folgend, Zahlungen seit Jahren unterhalb eines anerkannten Existenzminimums gewährt (vgl. Transfer-Enquête-Kommission 1981). Die geltenden Regelsätze decken weniger den tatsächlichen Bedarf im Sinne einer Mindesteinkommensregelung, sie stecken vielmehr die unterste Einkommensgrenze der Armutsbevölkerung ab.

1984 erhielten im Jahresverlauf schon 2,6 Mio. Menschen Sozialhilfe (SH), fast jeder zweite regelmäßige Empfänger war jünger als 25 Jahre (vgl. Frankfurter Rundschau vom 22.4. 86). Der Anteil der tatsächlich Betroffenen dürfte noch um einiges höher liegen: Es wird davon ausgegangen, daß etwa auf jeden Sozialhilfebezieher ein ebenfalls Anspruchsberechtigter obne staatliche Unterstützung kommt. Die Rentenversicherung zwingt (1982) mehr als 5,7 Mio. Rentner mit unter $1000 \mathrm{DM}$, darunter über 500000 sogar mit unter $600 \mathrm{DM}$ monatl. auszukommen (vgl. Opielka 1985, S. 155). Da 1985 nur noch $36 \%$ der registrierten (!) Arbeitslosen Arbeitslosengeld (ALG) erhielten, ist ein absolut und relativ wachsender Teil der Betroffenen auf den Bezug von Leistungen nach dem BSHG oder auf materielle Versorgung unter den Bedingungen der Arbeitslosenhilfe(ALH) angewiesen und damit den mit beiden verbundenen Abhängigkeiten von Familienangehörigen ebenso ausgeliefert, wie den materiellen Restriktionen der Leistungsgewährung. Darüber hinaus führt der Ausgrenzungsdruck des dreigliedrigen Sicherungssystems zu einem bedrohlichen Anwachsen von Erwerbslosengruppen obne jegliche Unterstützungsansprüche (vgl. Balsen u.a. 1984). Sozialpolitisch versagt das auf die Bedingungen der 60er Jahre adjustierte Sicherungssystem also schon seit Jahren - weder sichert es auf Dauer Einkommen, noch verhindert es wenigstens Armut: Verschiedene Schätzungen weisen zwischen 4 und 11,5 Mio. Personen aus, die von materieller Armut betroffen sind (vgl. Balsen u.a. 1984; Leibfried/Tennstedt 1985 a; Gerhardt 1985; Adamy/Steffen 1984).

Erweist sich die Orientierung an der individuellen Lohnhöhe also unter Leistungsgesichtspunkten als unzureichend, so gilt dies zunehmend auch für den Finanzierungsaspekt. Im bis- 
herigen System ist die zyklische Leistungsreduktion mit Strukturnotwendigkeit eingebaut: Je weniger »Arbeitsplatzbesitzer « immer mehr Arbeitslosen gegenüberstehen, desto mehr verfehlt eine lohn- und gehaltsfinanzierte Existenzsicherung ihre grundlegende Aufgabe, zumal in aller Regel individuelle Bedarfs- kollektiven Finanzierungsaspekten untergeordnet werden. Wie in einem kybernetischen Regelkreis manifestiert sich nahezu ungebrochen das vermeintliche sozialpolitische »Naturgesetz«als Anpassung des sozialen Leistungspegels an ein konjunkturabhängiges Finanzvolumen. Statt in diesem Prozeß einen wirklichen armutsverhindernden Leistungsstandard als Parameter konstant zu halten und Wirtschafts- und Sozialpolitik an diesem Niveau auszurichten, werden umgekehrt die Leistungen des Sozialsystems zur abhängigen Variable, an der die Finanzierungsschraube ansetzt.

In dem Maße, in dem Arbeiter- und Armenpolitik sich auch in ihrem materiellen Gehalt (ihrer Funktion nach sind beide schon seit den Anfängen der Sozialpolitik identisch) wieder annähern, werden neue Formen der Partizipation am bzw. der Verteilung des gesellschaftlichen Reichtums sogar immanent zur Notwendigkeit: Tatsächlich erforderte eine am Bedarf orientierte Mindestsicherung (Grundsicherung) einen steuerfinanzierten Verteilungsmodus auf garantiertem Niveau. Dies gilt besonders deshalb, weil die gegenwärtige Krise des Beschäftigungssystems das Ergebnis eines langanhaltenden, strukturellen Prozesses ist, der mit altbekannten Methoden weder kurz- noch mittelfristig in den Griff zu bekommen ist. Der gegenwärtige Strukturwandel der (Erwerbs-)Arbeitsgesellschaft im Zeichen der Reorganisation der Kapitalverwertungsbedingungen wird u.a. an zwei, die Arbeiterexistenz zentral berührenden Ent wicklungen offensichtlich. Die sich zuspitzende Tendenz der Polarisierung der Lohnarbeit (für die Industriearbeit vgl. Kern/Schumann 1985; für die Büroarbeit vgl. Baethge/Overbeck 1986) in wohlsituierte Kern- und labile Randbelegschaften erhält - neben wichtigen technischen Imperativen - starke Impulse aus dem Wandel der Bedingungen des Verkaufs der Arbeitskraft in der letzten Dekade, dem Anwachsen und der zeitlichen Konstanz der industriellen Reservearmee. Wenn auch der tatsächliche Umfang der Arbeitsplatzeffekte »neuer « Technologien noch nicht valide zu bestimmen und zugleich auch die Bedeutung demographischer Faktoren für das Ausmaß der Arbeitslosigkeit zu berücksichtigen ist, so spricht doch vieles dafür, daß sich die Schere zwischen Produktivitätsent wicklung und Wirtschaftswachstum noch vergrößern wird, und daß ohne einschneidende wirtschafts- und tarifpolitische Maßnahmen das gesellschaftliche Leben weiterhin von den Bedingungen der Massenarbeitslosigkeit diktiert sein wird. Ein entsprechender sozialpolitischer Handlungsbedarf wird von »Vordenkern« in allen politischen Lagern erkannt. Den einen geht es dabei um das langfristige »Einrichten « auf die vorliegenden Strukturdaten im Sinne einer Optimierung der Akkumulationsbedingungen; den anderen geht es um die Bewältigung der Beschäftigungskrise und um die Errichtung eines stabilen Sozialsystems, bzw. z.T. darüber hinaus, um die Nutzung relevanter Potenzen in einer historisch besonderen Situation von für enorm gehaltenen Produktivitätssteigerungen. Alle aber setzen bei dem Gedanken an eine gesellschaftliche Subsistenzgarantie für die Subjekte an. 


\section{Entgegengesetzte Zielperspektiven}

\section{Substistenzgarantie und mmarktgerechtes« Sozialsystem}

Das Konzept eines garantierten Mindesteinkommens (ME) wird seit seinem Entstehen im Namen der Überwindung materieller Armut diskutiert. Wenn konservative und liberale Strategien auch mit diesem Anspruch auftreten, so interessiert sie das Instrument doch vorrangig aus anderen Gründen. Die entsprechenden Vorschläge ${ }^{1}$ zur "Sozialdividende « und zur »negativen Einkommensteuer (NES) zeichnen sich dadurch aus, daß sie die Gewährung eines Mindesteinkommens in der Regel alternativ zur Gesamtheit der Leistungen des "Sozialstaats« verstehen: Das vielgliedrige System sozialer Sicherung soll auf eine Transferleistung reduziert und zugleich sollen Steuer- und Transfersystem integriert werden. (Auch ein Teil der nicht-materiellen sozialstaatlichen Aufgaben läuft so Gefahr, auf Finanzämter übertragen bzw. an Bankschalter verwiesen zu werden.) Eine solche ökonomistische Effektivierung des Transfersystems würde die z.T. enormen Verwaltungskosten des "Sozialstaats« minimieren, während das zumeist an der Armutsgrenze (oder sogar darunter) orientierte Niveau der Transfers »überzogene Ansprüche« abbauen würde.

An die Seite dieser antizipierten Senkung der Staatsquote (Fluchtpunkt aller markttheoretischen Überlegungen) treten aber zumeist auch arbeitspolitische Ziele. So wird das System der NES von einigen seiner Promotoren explizit als »Arbeitsanreizsystem « begriffen: die Monetaristen haben dieses System sogar auf der Suche nach Steuerungsinstrumenten zur Erhöhung des Arbeitsangebots ent wickelt. ${ }^{2}$ Ein erhöhtes Angebot auf dem Arbeitsmarkt soll natürlich auch flexible Reaktionen des Preises der Arbeit bewirken. Ein exakt darauf abgestimmtes Verfahren der gewichteten Anrechnung von Arbeitseinkommen auf das Sozialeinkommen soll die Arbeitsaufnahme auch bei niedrigem Preis individuell »lohnender " gestalten, als bei den gegenwärtigen Sicherungssystemen, die Leistungen nur kausal und in der Regel alternativ zu Arbeitseinkommen gewähren. $» D i e$ heimlichen Ziele der konservativen Vorschläge zu einem Recht auf Einkommen ... (sind, F.M.), das Arbeitsangebot für schlecht bezahlte und stark belastende Tätigkeiten zu erhöhen und einen Großteil der bisherigen sozial-staatlichen Ausgaben den Unternehmen faktisch als Subvention zur Verbilligung ihrer Lohn- und Gehaltszahlungen zukommen zu lassen...« (Bust-Bartels 1985, S. 32). Daneben wird auf einer grundsätzlicheren Ebene mit einem ME die Hoffnunf auf eine systemadäquate Optimierung der »Marktfähigkeit « der lebendigen Arbeit in der Tradition der klassischen Arbeitsmarkt- und Wirtschaftstheorie verbunden. Letztere bedingt, daß jede Tauschhandlung freiwillig erfolgt; die geschichtliche Inthronisierung des Lohnarbeitszwangs (Unterbindung aller Lebensperspektiven jenseits des Lohnarbeitsverhältnisses) verhindert jedoch seit dessen Erzwingung (vgl. Vobruba 1985, S. 44 f.; Dießenbacher 1985, S. 14 ff.) diefreie Wahlmöglichkeit der Besitzer der »fiktiven Ware « Arbeitskraft zwischen Verkauf und Enthaltung, zwischen Erwerbstätigkeit und Freizeit. Die Modelle, die dem »Faktor« Arbeit ideologisch verbrämt und modelltheoretisch notwendig schon immer diese Wahlmöglichkeit unterstellen, ignorieren den

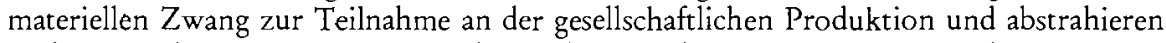
mithin von den strukturellen Gewaltverhältnissen der kapitalistischen Produktion. Kein Wunder also, daß neoklassische Ökonomen in einem ME nichts anderes als eine Effektivierung des Marktgeschehens sehen, d.h. eine weitere Annäherung der Wirklichkeit an das Modell (»reiner Arbeitsmarkt «). Anzumerken bleibt aber, daß, selbst wenn man Stimmigkeit für den Rest der Überlegungen annehmen könnte, ein Subsistenzminimum auf oder unter dem 
Niveau der heutigen Sozialhilfe kaum in der Lage sein dürfte, die Erwartungen zu erfüllen; denn erst ein wirklich bedarfsdeckendes ME - das ein Haushaltseinkommen in der Nähe heutiger Durchschnittslöhne garantieren müßte - würde die notwendigen Voraussetzungen für ein »marktgerechtes« Handeln der Subjekte im Sinne einer real existierenden Wahlmöglichkeit zwischen Teilnahme am (Lohnarbeit) und Abstinenz vom (Eigenarbeit/Freizeit) Arbeitsmarkt schaffen. Erst jenseits der Sicherung der Reproduktion der Arbeitskraft werden essentielle Wahlmöglichkeiten realistisch.

In der Bundesrepublik werden ME-Garantien häufig im Kontext konservativer Modernisierungsstrategien reffektiert. So wird etwa zur Realisierung der konservativ-liberalen Dauerforderung der Minimierung der Lohnkosten neuerdings eine fundamentale Umstrukturierung des »Sozialstaats« vorgeschlagen: Die Kosten der sozialen Sicherung sollen von den »Arbeitskosten « ent koppelt (Kommission Zukunftsperspektiven ... 1983), das »Arbeitsverhältnis behutsam vom Sozialverhältnis getrennt« werden (Biedenkopf 1984). Während E. Albrecht (1983) und K. Biedenkopf das geänderte Konzept sozialer Sicherung über eine Erhöhung der Mehrwertsteuer finanziert sehen wollen, um das staat liche Sozialversicherungssystem in der Tendenz in einer einzigen, niedrigen Transferleistung aufgehen zu lassen, favorisiert der Kronberger Kreis (1984) die NES (vgl. auch Engels u.a. 1974; Molitor 1973, 1981; Pabst 1983; Miegel 1981; Dahrendorf 1983). - Dieses Modell der Reform des Sozialsystems fügt sich in die ebenfalls von dieser Seite vorgetragenen Konzepte der Ökonomisierung weiterer Lebensbereiche ein. Auch die seit längerem in CDU und FDP diskutierten Modelle der Grundrente (z.B. Biedenkopf, Bangemann, Fink) oder Sockelrente (Mischnick) zielen auf die(Teil-)Privatisierung der Sozialleistungssysteme. Während also auf der einen Seite die Lohnkosten um einen Großteil der Lohnnebenkosten reduziert werden sollen, werden auf der anderen Seite über die staat liche Mindestsicherung hinausgehende Sicherungsbedürfnisse auf den privaten Kapitalmarkt verwiesen; der »reine Arbeitsmarkt« wird um ein »marktgerechtes Sozialsystem« ergänzt.

An diese ökonomistische "Zurichtung" des Transfersystems knüpfen konservative Theoretiker aber auch ordnungspolitische Überlegungen, die die Krise der (Erwerbs-)Arbeitsgesellschaft in besonderer Weise reflektieren: Die Spaltung der Gesellschaft soll individuell erträglich und gesellschaftlich effektiviert gestaltet werden. Das so auf niedrigem Niveau formulierte Recht auf Einkommen wird folgerichtig alternativ zum Recht auf Arbeit diskutiert - konservative Sozialpolitik wird final auf seine sedativen Wirkungen reduziert. Das Angebot ist eindeutig: Für den Verzicht auf gesellschaftliche Mitbestimmung (politische Desintegration) und kompensativ für die Verweigerung der Teilhabe an gesicherten und tradierten Vergesellschaftungsformen (»produktive« Desintegration) wird für einen Teil der Bevölkerung die Gewährung einer mehr oder minder restriktionslosen Sozialhilfe (materielle Teil-Integration) erwogen. Dafür sind aufgeklärte konservative Theoretiker bereit, die Arbeitspflicht weiter zu relativieren und mit der Leistungsideologie insoweit zu brechen, daß sie die unbedingte Verantwortung der (Arbeits-)Gesellschaft für die Folgen ihres Wirtschaftens im Subsistenzbereich akzeptieren.

Entgegen allen aktuellen Tendenzen des Sozialabbaus ist nämlich eine Entflechtung des »Nexus von Arbeiten und Essen «(Vobruba) nicht nur von der Höhe des Sozialprodukts her möglich: eine solche Relativierung der »bedingten« Arbeitspflicht läßt sich auch in eine historische Entwicklung einreihen (vgl. Vobruba 1985). In der heutigen Phase der Entwicklung des Kapitalismus geht es weder um die Instrumentalisierung des Hungers zur Ausbildung und Institutionalisierung (1. Phase), noch zur Aufrechterhaltung(2. Phase) des Arbeitsmarktes oder 
um die Internalisierung einer Arbeitsmoral durch gezieltes Staatshandeln. Die fast ungebrochen leistungsideologischen Imperativen gehorchende Reproduktion hierarchischer Strukturen des Erwerbssektors, (auf niedrigerem Niveau) auch innerhalb der sozialen Sicherungssysteme, erweist zwar auch zu Krisenzeiten ihre disziplinierenden Qualitäten; diese Form der individuellen Disziplinierung wird aber in dem Maße verzichtbar, wie gesellschaftlich diese Funktionen von einer stabilen industriellen Reservearmee übernommen werden. Im Zeichen eines hohen Niveaus an verdinglichten Bedürfnis- bzw. Konsumtionsstrukturen, die ebenfalls diese Funktionen übernehmen können, wird es möglich, begrenzte Lebensperspektiven außerbalb der Lohnarbeiterexistenz zuzulassen. Wenn damit höherrangige Herrschaftsfunktionen abgesichert werden, braucht also eine Subsistenzgarantie (selbst eine bedeutendere als heute) nicht dysfunktional zu werden. G. Vobruba (1985, S. 42 ff.) gelangt durch eine Analyse von vier Stadien des Bedeutungswandels der Armut und ihrer je spezifischen Funktionalität für bestimmte Entwicklungsphasen des Kapitalverhältnisses überzèugend zu der Annahme, daß es aus dieser Entwicklung heraus plausibel sei, wenn die bestehende Phase der »bedingten Entflechtung von Arbeit und Essen « von einer »unbedingten Entflechtung « abgelöst werde.

\section{Garantierte Grundsicherung in allen Lebenslagen}

Diese Entflechtung von »Arbeiten und Essen« wird von Theoretikern des links-alternativen Spektrums durch den Gedanken an ein garantiertes ME in einer anderen Perspektive aufgegriffen. Die verschiedenen Überlegungen stützen sich u.a. darauf, daß diese Entflechtung als gesellschaftliche Tendenz ja schon einige Zeit wirksam ist: Massenarbeitslosigkeit bedeutet gesamtgesellschaftlich eine sich anarchisch durchsetzende strukturelle Form der Arbeitszeitverkürzung; ein anwachsender Teil der Bevölkerung ist dauerhaft zur Deckung seines Lebensunterhalts auf Transferzahlungen angewiesen. Obwohl die Neu-Konstruktion des sozialen Sicherungssystems also auch aus immanenten Gründen (s.o.) zur Notwendigkeit wird, ist die entscheidende Frage jedoch die, wie sich die Entflechtung von Arbeiten und Essen gesellsschaftlich durchsetzen wird; ob anarchisch über die Marktgesetze oder "gesellschaftspolitisch kalkuliert und kontrolliert « (Vobruba 1986, S. 39). In diesem Sinne gehört es zu den wichtigen Aufgaben einer sozial verantwortlichen Politik, dieses Politikfeld zu besetzen und Gestaltungsperspektiven zu entwickeln. Tatsächlich könnte die hier diskutierte weitere Lockerung des $Z_{\text {wangsverhältnisses von Erwerbsarbeit und Reproduktion emanzipative Po- }}$ tenzen freisetzen, wenn es gelänge, eine radikale Arbeitsumverteilung durch Arbeitszeitverkürzung mit der Garantie einer ausreichenden materiellen Sicherung der Existenz für alle Bevölkerungsteile zu verbinden. Statt einer weitergehenden Differenzierung klassen-, aber v.a. schichtspezifischer Interessenlagen, könnte durch eine solche Garantie zur aktiven Teilhabe an der produktiven und sozialen Gestaltung der Gesellschaft eine harmonischere soziale Entwicklung bewirkt werden, da unzweifelhaft eine in ihrer Wirkung außer Kraft gesetzte industrielle Reservearmee gesellschaftliche Spaltungstendenzen mindern würde.

Schon hier wird deutlich, daß auch im links-alternativen Umfeld das garantierte ME nicht nur als einfache sozialpolitische Maßnahme diskutiert wird. Der direkte Bezug zu einer möglichen Änderung materieller Reproduktionsbedingungen weckt im Lichte der unterschiedlichen Konzepte hochgesteckte Erwartungen. Während ein Teil der Diskutanten die Frage zu beantworten sucht, wie mittels einer alternativen Sozialpolitik den sich abzeichnenden Spaltungstendenzen entgegengewirkt werden kann und welche Aufgabe einem ME dabei zukom- 
men könne, wird die Idee des ME einem anderen Strang der Diskussion zum »reformpolitischen Hebel schlechthin «(Bust-Bartels): einem Teil der Autoren geht es um nicht weniger als die Umkehr der Vorzeichen der gesellschaftlichen Entwicklung.

(a) So wird an das verteilungspolitische Instrument ME natürlich die Anforderung gestellt, das Sozialsystem dauerhaft zu stabilisieren und zur Überwindung materieller Armut in einer fortgeschrittenen Industriegesellschaft beizutragen. Die Utopien reichen aber darüber hinaus von der Förderung selbstorganisierter, alternativer Wirtschaftsformen, dem Anwachsen von »Eigenarbeit« und »Selbsthilfe« zu ungunsten des Anteils der Erwerbsarbeit (u.a. Gerhardt/Weber 1984; Schmid 1984; Gretschmann 1985; Huber 1982; Offe 1984; Gorz 1983; Kollektiv Charles Fourier 1985) bis hin zur Überwindung der Lohnarbeit und damit der Grenzen des kapitalistischen Systems (v.a. Opielka 1985; Gorz 1983). ${ }^{4}$

(b) Gleichzeitig wird daran anschließend die Bedeutung eines ME im Kontext einer grundsätzlichen Kritik der Werte der Arbeitsgesellschaft betont.

Das strategische Konzept der Vergangenheit, umverteilungspolitische Vorhaben über zu erwartendes Wirtschaftswachstum und der damit verbundenen Steigerung der Produktion zu finanzieren, läßt nicht nur Form und Relation der Klassenreproduktion unangetastet, es setzt ebenso ungebrochen alle Hoffnung auf eine Produktionsweise, die im Zeichen der Dialektik von Produktion und Destruktion die Strukturgesetze einer ökonomistischen Form technologiegestützter Rationalität perpetuiert und deshalb zunehmend als kontraproduktiv erkannt wird: Die sozialen und ökologischen Kosten der traditionell-industriellen Produktion drohen selbst bei einer »geläuterten « Wachstumsökonomie ins Unermeßliche zu wachsen. Deshalb soll neben dem sozialen, der ökologische Umbau der Gesellschaft gleich mitvollzogen werden. Die Befürworter sehen im ME eine Maßnahme, die in der Lage ist, auf beiderlei Bereiche gleichermaßen zu wirken. ${ }^{5}$ Durch die Basissicherung sollen die materiellen Bedingungen struktureller Gewaltverhältnisse soweit variiert werden, daß essentielle Freiräume entstehen, die es den Subjekten erlauben, sich einem »ökologischen Paradigma« (Opielka) entsprechend zu verhalten. Zumeist im Kontext unterschiedlicher dual wirtschaftlicher Konzepte wird das Schwergewicht der Untersuchungen auf sich ausweitende Wahlmöglichkeiten und Aktivitäten im informellen Sektor (»Sphäre der Autonomie«, Gorz) gelegt, während die Rigidität bestehender Strukturen häufig nicht angemessen gewürdigt wird; der Ansatz von Gorz (1980; 1983) gründet geradezu darauf, den heteronomen gesellschaftlichen Bereich von Freiheitsansprüchen auszunehmen. - Letztlich werden unzulässigerweise die erhofften gröBeren Entscheidungsspielräume der Subjekte mit der Überwindung der produktivistischen Logik des Industriesystems gleichgesetzt, während die Frage unbeant wortet bleibt, ob eine höhere Einkommensgerechtigkeit wirklich zugleich die Überwindung der »Privatheit der Interessen « und ein ökologisches Handlungskonzept hervorrufen kann, oder ob es sich dabei nicht vielmehr um eine Projektion des Gesellschaftskonstrukteurs als um ein erwartbares mehrheitliches Handlungsmuster handelt. ${ }^{6}$

(c) Neben seiner Lohnarbeitszentriertheit und dem immanenten Vertrauen auf produktivistische Rationalität ist das soziale Sicherungssystem auch Ausdruck und stützendes Moment der patriarchalen Struktur der Gesellschaft: in seiner Ehezentriertheit subventioniert es eine spezifisch bürgerliche Lebensform, verfestigt deren Alltagsideologien und ist materielle Barriere für die weibliche Emanzipation.

Orientiert an der Biographie des Mannes (als »Haushaltsvorstand« und »Ernährer«) bestärkt das Transfersystem die hierarchischen Beziehungen in der Familie, indem durch die Bedingungen der Leistungsgewährung der Frau ökonomische Unabhängigkeit verwehrt und Ten- 
denzen zur Selbstbestimmung erschwert werden (vgl. Blickhäuser/Molter 1986; Schreyer 1986, S. 162 ff.). Um dieser Situation zumindestes im materiellen Bereich gerecht zu werden, wird die Forderung nach einem garantierten ME mit der Forderung nach Individualbezug statt Haushaltsbezug und Ehegattensubsidiarität verbunden (Opielka 1985), um die »Ehe als Versorgungsinstanz gegenstandslos«(Blickhäuser/Molter 1986, S. 111) werden zu lassen und um »die patriarchalische Trennung zwischen bezahlter und unbezahlter Arbeit aufzuweichen « (ebd.). Ein bedarfsorientiertes Kinder- und Erziehungsgeld soll zusätzlich ökonomische Abhängigkeiten bei Kindererziehung vermeiden helfen (vgl. Die Grünen 1986; Arbeitskreis »Sozialpolitik « 1986); ehebezogene Leistungen (z.B. Familienlastenausgleich) sollen in kinderbezogene Leistungen umgewandelt werden. M. Opielka faßt das Fernziel dieser Forderung zusammen: „Sollen die Frauen gleichberechtigt sein, so muß die gesamte Arbeit umverteilt werden und müssen die gesamten Einkommen in den Umverteilungsprozeß einbezogen werden.« (1985 c, S. 66)

Dieser kurze Überblick zeigt, daß ein Großteil der gegenwärtig das links-alternative Spektrum bewegenden politischen Themenbereiche in der einen oder anderen Weise mit der eigentlich verteilungspolitischen Forderung nach materieller Basissicherung verknüpft werden. Tatsächlich scheint durch die ME-Diskussion ein innovativer Prozeß in Gang gesetzt worden zu sein, dem es in einigen Bereichen gelingt - quer zu den Diskussionszirkeln -, sachbezogen Reformperspektiven zu erörtern: Schon in der Vergangenheit zentrale Forderungen wurden aus verkrusteten Diskussionszusammenhängen gelöst und werden mit entsprechender Akzentuierung neu thematisiert vorgetragen. Sicherlich hat auch das Aufgreifen der ME-Forderung dazu geführt, daß die Auseinandersetzungen um eine emanzipatorische Politik jenseits der sich an ausgetretenen Pfaden orientierenden Theoriediskussion der 70er Jahre neu belebt worden sind (vgl. aber auch Gorz 1980).

\section{Die Struktur der Modelle}

Der Klarheit der ME-Forderung ist es allerdings kaum dienlich, wenn durch die Breite der Diskussion und durch unterschiedlichste Inhalte und Zwecksetzungen die Grenzlinien zwischen den einzelnen Perspektiven versch wimmen; wenn nur schwer offenbar wird, daß sich im Kontext einer verteilungspolitischen Maßnahme mebrere reformpolitische Diskussionsansätze gleichzeitig gegenüberstehen. ${ }^{7}$ An die Seite dieser perspektivischen Uneindeutigkeit der Forderung tritt darüber hinaus auf den ersten Blick häufig eine politische Indifferenz: Zumindest in den Anfängen der jüngeren ME-Debatte wurde überwiegend modelltheoretisch argumentiert, was im Ergebnis mehr Ähnlichkeiten und Übereinstimmungen zwischen den einzelnen Ansätzen, aber v.a. zwischen alternativen Transformations- und neokonservativen Modernisierungsmodellen suggerierte, als sie den Inhalten und Zielen nach tatsächlich berechtigt sind. Durch diese anfängliche Konzentration auf eine Modelldiskussion wurde u.a. versucht, mit Blick auf konservative Bündnispartner die Gemeinsamkeiten mit diesen zu betonen, während aber z.T. entscheidend differierende gesellschaftstheoretische Implikationen der Ansätze, die weit über einzelne finanzierungstechnische Parallelen hinausgehen, verdrängt wurden.

Durch die enorme Ausweitung, die die Diskussion um eine materielle Basissicherung in den letzten drei Jahren erfahren hat, liegen mittlerweile überaus differenzierte Betrachtungen vor. Schon eine punktuelle Beschäftigung mit den im einzelnen jeweils sehr unterschiedlichen Entwürfen zur Sozialdividende von: Rhys-Williams (1953), Roberts (1986), Gretsch- 
mann $(1985,1985$ a), Opielka $(1984,1985,1986)$, Kollektiv Charles Fourier (1985) und im weiteren Sinne auch Gorz $(1983,1985)$ oder zur negativen Einkommenssteuer (NES) von: Friedman (1984), Engels u.a. (1974), Kronberger Kreis (1984), Almsick (1981), Wiemann (1985), Gerhardt/Weber (1984) und Vobruba $(1985,1986)$ könnte zeigen, wie wenig das gleiche Transfermodell mit der entsprechenden gesellschaftstheoretischen Intention der einzelnen Autoren zu tun hat. Parallelen zwischen den Beiträgen finden sich eher auf der Ebene der sie leitenden Gesellschaftsanalysen, als auf der Modellebene. Da aber gleichzeitig die strukturellen Wirkungen der unterschiedlichen Umverteilungsmaßnahmen immer wieder unterschätzt werden, ist es dennoch notwendig, zunächst auf den strukturellen Gehalt der Vorschläge einzugehen.

Bei insgesamt vier verschiedenen Transfermodellen stehen in der aktuellen Debatte zwei Konzepten eines Mindesteinkommens zwei Grundeinkommensmodelle gegenüber ${ }^{8}$ :

1) Die weitestgehenden Vorschläge von G. Rehn (1973) und G. Adler-Karlsson (1979) verbinden in der Nachfolge von J. Popper-Lynkeus (vgl. dazu Bielke 1978), aber auch des frühen Fabier-Sozialismus (vgl. dazu Gretschmann 1981) ökonomische Mehrsektorenkonzepte mit einer Grundeinkommensgarantie. Eine zeitlich begrenzte Arbeitspflicht im gesellschaftlich organisierten »Grundbedarfssektor (Adler-Karlsson) begründet zugleich ein lebenslanges Versorgungsrecht mit entsprechenden Gütern, während in der verbleibenden Zeit selbstbestimmten Tätigkeiten nachgegangen oder sich an der privat wirtschaftlich-kapitalistischen Produktion (»Luxusgüter «) beteiligt werden kann. Durchaus in dieser Tradition einer sektorisierten Ökonomie, aber mit einem anderen Schwerpunkt ist die Position von A. Gorz $(1983,1985,1985$ a, 1986) zu sehen: Gorz geht es um die Garantie eines »lebenslänglich gesicherten Volleinkommens« (1985 a), das im Vergleich zu anderen Ansätzen gerade nicht auf der "Entkopplung von Arbeit und Einkommen «, sondern auf der Entkopplung von "Einkommen und Arbeitszeit « beruht. Mit diesem Übergang zur Lebensarbeitszeit (Vorschlag: 20000 Stunden pro Leben bzw. 10 Jahre Vollarbeitszeit; 1983, S. 66 ff.), deren schrittweise Reduktion an der Produktivitätsentwicklung des heteronomen Sektors (traditionell kapitalistische Produktion) orientiert werden müßte, würde bei voller Zeitsouveränität das Recht auf Arbeit mit dem Recht auf angemessenes Sozialeinkommen verbinden, während gleichzeitig drastische Arbeitszeitverkürzungen immer größere Autonomiebereiche erwarten ließen. Das antizipierte Anwachsen der Tätigkeiten im 'selbstbestimmten' (autonomen) Bereich soll sodann in der Tendenz Bedingungen gewährleisten, die es erlauben, den heteronomen Herrschafts- und Produktionsapparat zurückzudrängen. Die Frage der dazu notwendigen politischen Souveränität wird bei Gorz allerdings ausgeklammert. Die Gesellschaftsutopie von Gorz setzt also bei einer gesellschaftlich organisierten Arbeitsumverteilung ohne Realeinkommensverluste an (ohne allerdings auch in den Produktionsapparat eingreifen zu wollen), wohingegen Rehn und Adler-Karlsson den Grundbedarfssektor einer gesellschaftlichen Planung unterstellen und nur den Subsistenzbereich garantieren wollen. Die Zielperspektiven dürften aber in einer sehr ähnlich strukturierten 'Postkapitalist ischen Gesellschaft' zu suchen sein.

2) Demgegenüber baut das zweite Grundeinkommensmodell nicht gleich auf einem geänderten Wirtschaftssystem auf. Die Sozialdividende (auch Bürgergehalt oder Nationaldividende genannt) zielt über eine Integration von Steuer- und Transfersystem auf die Garantie eines Grundeinkommens, indem jedem Bürger völlig vorbehaltlos regelmäßig ein bestimmter Betrag zur Sicherung seiner Grundbedürfnisse überlassen wird. Eine direkte Bedürftigkeitsprüfung wird so umgangen; ob Bedürftigkeit vorgelegen hat oder nicht, stellt sich erst am Jahres- 
ende heraus, wenn das Grundeinkommen zusammen mit allen anderen Einkommensarten von den Finanzämtern veranlagt wird.

Die Modelleinnahmen unterscheiden sich v.a. in der Höhe des zur Teilhabe am gesellschaftlichen Leben für notwendig angenommenen Garantiebetrags (Vorschläge reichen von 500 DM [Gretschmann 1985 a] bis zu 1600 DM [Blickhäuser/Molter 1986] monatlich) und in der Frage, ob diese staatliche Umverteilung alternativ zum herkömmlichen System sozialer Sicherung oder zusätzlich dazu zu konzipieren sei. M. Opielka (vgl. 1985, S. 146 f.) will z.B. nur die schon heute steuerfinanzierten Bereiche des Sozialleistungssystems vom Grundeinkommen abgedeckt wissen; die traditionellen Sozialversicherungen sollen weiterhin die Sicherung des durch Erwerbsarbeit finanzierten Lebensstandards übernehmen.

3) Auf der folgenden Modellebene unterscheidet sich die negative Einkommensteuer von der Sozialdividende nur unwesentlich in einigen finanzierungstechnischen Aspekten. Die Subsistenzgarantie wird hier über ein festgelegtes Mindesteinkommen eingelöst, das jedem aus dem Kreis der Berechtigten ${ }^{9}$ zusteht, der über kein Einkommen verfügt. Wie die Sozialdividende wird auch die NES in der Mehrzahl als arbeitsunabhängige Leistung diskutiert. Allerdings wird - abhängig vom entsprechenden, meist linearen (!), Steuersatz - Erwerbstätigkeit in der Weise "belohnt «, daß die Grenze des verfügbaren Einkommens, auf die geringes Erwerbseinkommen um Bruchteile des Mindesteinkommens aufgestockt wird, mit eben diesem Erwerbseinkommen bis zu einem bestimmten Punkt wächst. Umgekehrt betrachtet heißt das, daß die Höhe staatlicher Leistungen (»negativer« Steuern) mit zunehmendem Erwerbseinkommen kontinuierlich abnimmt, bis der Prozeß umschlägt und die Höhe des Einkommens zur Zahlung von positiven Steuern (»negativen Transfers«) verpflichtet. Durch die Höhe des Steuersatzes wird gewährleistet, daß jeweils nur ein Teil des Erwerbseinkommens auf die staatlichen Transfers angerechnet wird, so daß bei Arbeitsaufnahme die verfügbaren Einkommen steigen, ohne bei nur geringem Arbeitsumfang bzw. geringer Bezahlung um die gesamten Transfereinkommen gekürzt zu werden. Die Übergänge zwischen Erwerbstätigkeit und Nicht-Erwerbstätigkeit werden auf diese Weise fließend gestaltet, die Arbeitsaufnahme wird im unteren Einkommensbereich materiell »lohnend " gemacht. Die Sozialdividende wirkt in strukturell gleicher Weise. Im Rückblick gestalten sich die verfügbaren Einkommen bei beiden Modellen der Höhe nach identisch (vgl. Gerhardt/Weber 1984, S. 29 ff.), während aber die Sozialdividende als »Bruttorechnung « eine gigantische Vorfinanzierung neben dem z.T. an sich schon beträchtlichen Umfang an Transferzahlungen erfordert.

4) Das zweite Mindesteinkommenskonzept setzt auf die »Sockelung des Sozialsystems". Diese Forderung, die bestehenden Sozialsysteme in ihrer Gesamtheit durch einheitliche Sockelbeträge so zu untermauern, daß in der Tendenz ein garantiertes ME in allen Lebenslagen verwirklicht wird, ist als Alternative zu den oben diskutierten Modellen zu verstehen: sie faßt aus der Perspektive eher traditioneller Sozialpolitik deren Kritik und Ansätze zur Weiterentwicklung der bereits bestehenden Rudimente einer Mindestsicherung zusammen. Gemeinsam ist dieser Gruppe von Vorschlägen die Ablehnung des bedingungslosen (blinden) Transferautomatismus der Mehrzahl der Vorschläge zur Sozialdividende und zur NES, sowie die Absicht, strukturtransformierende Einsichten aus der Kritik bestehender Leistungsformen zu gewinnen und sich nicht auf das Vabanquespiel der Installation eines grundlegend anderen (und letztlich in der Wirkung ungewissen) Umverteilungssystems einzulassen (vgl. Hanesch 1984; ders./Klein 1986; Hauser u.a. 1981; Bust-Bartels 1985, 1985 a; Leibfried 1986; TransferEnquête-Kommission 1981). Als einzelne Kennnzeichen der "Sockelungsperspektive« sind zu nennen: 
- Die Konzentration auf die sozialpolitische Bedeutung des Prinzips der Bedarfsdeckung, das erhalten bzw. infolge seiner Destruktion durch die "Sparpolitik« der letzten Jahre erst wieder zur Geltung gebracht werden muß (vgl. Leibfried/Tennstedt 1985 a).

- Die unbedingte Priorität des Bedarfsprinzips - das als Garant für ein umfassendes existentielles Teilhaberecht gestaltet werden soll -, gegenüber dem in der Sozialversicherung als Ausdruck leistungsideologischer und ausgrenzender Herrschaftsfunktionen dominierenden Äquivalenzprinzips, das die ungleichen Reproduktionsbedingungen des Erwerbssektors für den Einflußbereich der Sozialversicherung kopiert: Die unheilvolle Allianz aus Äquivalenzund Versicherungsprinzip (»leistungs- und beitragsbezogene Versicherungsleistungen«) für den unteren Einkommensbereich soll gebrochen werden.

- Nicht diegrundlegende Integration aller sozialen Sicherungssysteme nach einem Transfermodell, sondern die Integration von Mindestansprüchen in deren Teilbereichen ist Fluchtpunkt dieser Forderung; bei Bedarf sollen alle heute bestehenden Ansprüche steuerfinanziert auf dieses Mindestniveau »aufgestockt « werden.

All diese vier Umverteilungsmodelle sind allein ihrer Struktur nach nicht an ein bestimmtes Gesellschaftssystem gebunden; sie können sowohl widerspruchslos dem entwickelten Kapitalismus eingepaßt werden, als auch Transformationskonzepten dienen. Gerade dies macht das klassenübergreifende Interesse an ihnen aus! Allerdings ist die ME-Forderung nicht so »weltanschaulich uneindeutig«, wie M. Opielka und G. Vobruba (1986) meinen: Die materiellen Bedingungen der Reproduktion können ebenso wie ggf. die Struktur einer alternativen Reproduktionslogik nicht ohne Auswirkung auf die Klassenreproduktion bleiben.

\section{Das Mindesteinkommen in der Diskussion}

Die Notwendigkeit einer deutlich über dem heutigen Niveau liegenden garantierten Mindestsicherung dürfte auch weit über den Kreis der aktiv beteiligten Diskutanten hinaus außer Frage stehen; strittig ist allerdings, was mit dieser Forderung zusätzlich verbunden werden soll (oder kann), bzw. in welchen gesellschaftspolitischen Kontext die Forderung eingebettet werden muß, damit sie überhaupt wirksam werden kann. Da sich, wie oben schon angedeutet, die notwendige Differenzierung verschiedener »links-alternativer « Ansätze gerade nicht durchgängig auf der Modellebene vollziehen läßt, soll im Hinblick auf eine erst noch zu fühbrende Strategiediskussion auf die unterschiedlichen gesellschaftspolitischen Zielvorstellungen ebenso eingegangen werden, wie auf zu erwartende strukturelle Wirkungen der Vorschläge und auf strategische Aspekte einer möglichen Durchsetzung der Forderung.

\section{Die Qualität der Quantität}

Auch wenn die teilweise recht ungezwungene Diskussionshaltung in der ME-Debatte zeitweilig darüber hinwegtäuschen mag (wenn etwa »am grünen Tisch« mit riesigen Milliardenbeträgen jongliert oder wenn ein anderes Vergesellschaftungsmodell oder eine andere Reproduktionslogik konzipiert und durchgespielt wird) ${ }^{10}$, so muß sich doch für eine Beurteilung bei jedem Argumentationsschritt vergegenwärtigt werden, an welch zentraler Stelle die Überlegungen zu rütteln versuchen: Das ME ist eine Umverteilungsmaßnahme, jeder Realisierungsanspruch berührt augenblicklich Klasseninteressen. Die letztlich entscheidende Frage ist weniger, nach welchem Modell umverteilt wird, als vielmehr, ob der aktuelle Trend der 
Umverteilung "von unten nach oben « umgekehrt werden und ob dabei über die »Umverteilung innerhalb einer Klasse " (vgl. Costas 1985), die die Klassenreproduktion im wesentlichen unberührt ließe (Politik der 70er Jahre), hinausgegangen werden kann. Eine Beantwortung dieser Frage wird umgegangen, wenn man verdrängt, daß nicht das Verteilungsmodell, sondern dessen Umverteilungseffekte (im engeren Sinne die Höhe des ME) die Konfliktlinien zwischen den Klassenlagen ziehen. Rechnen kann man »rechts« ebenso gut wie »links«. Bei genauerem Hinsehen geht es im Kern also nicht um die Etablierung eines anderen Transfersystems/-modells, sondern um die gesellschafts-, wirtschafts- und sozialpolitische Ausrichtung insgesamt, d.h. um die Durchsetzungsfähigkeit hegemonialer Interessen: In welche Richtung können Verteilungsrelationen verändert werden. ${ }^{11}$ M. Greven (1986, S. 63) formulierte eine Prämisse, die häufig in der Diskussion auftaucht: »Ein 'Bürgergehalt', das unabhängig von einer Ermittlung von Lebenslagen und unabhängig von einem Versicherungsbeitrag als Rechtsanspruch besteht, bricht mit der herrschenden Systemlogik. « Doch wäre es ein verkürzender Ansatz, von dieser formalen Annahme allein - ohne Reflexion des materiellen Gehalts der Maßnahme - auszugehen. Das ME ist erst einmal nicht »systemsprengender « als andere Sozialeinkommen auch (vgl. Bleicher 1984, S. 76 f.);jeder Sozialtransfer stellt in sich eine Relativierung der Arbeitspflicht dar. Ganz offensichtlich ist die Höhe der Forderung ein entscheidendes Kriterium für deren Beurteilung und für den Grad der mit der Gewährung einhergehenden Diskriminierung oder »Freiheit «: Ein »Bürgergehalt « von z.B. 500 DM würde wohl systemstabilisierend zur Anwendung kommen; in der neokonservativen Variante stellt es durchaus keinen "Bruch" mit der Systemlogik, sondern vielmehr die Anpassungsfähigkeit des Spätkapitalismus unter Beweis.

Völlig unabbängig von der Form der Verteilung von Sozialeinkommen können (mit Hilfe von Aussagen zur Tendenz ihrer Wirkungen) unterschiedliche Quantitäten Auskunft darüber geben, welche Entwicklung mit der damit jeweils verbundenen Qualitätsänderung vermutlich eingeleitet wird:

- Je näher das ME-Niveau an die absolute Armutsgrenze heranreicht, bzw. je mehr Gruppen durch Vereinheitlichung des Sozialsystems mit reduzierten Ansprüchen konfrontiert und auf diese Weise ihren Lebensstandard im Bedarfsfall nach »unten « korrigieren müssen, desto mehr wird die Gültigkeit rein marktgesetzlicher Orientierungsmuster im Erfahrungsbereich eines jeden einzelnen ausgedehnt, werden die schon bestehenden Spaltungstendenzen in der Gesellschaft forciert und desto mehr wird einer weiteren »Amerikanisierung « der bundesrepublikanischen Wirklichkeit im Sinne neokonservativer Modernisierungskonzepte Vorschub geleistet.

Umgekehrt:

- Je mehr Transferzahlungen zu einem insgesamt nivellierenden Einkommensniveau beitragen, bzw. je weniger die individuelle Lebensführung vom Verkauf der Arbeitskraft abhängig ist, desto eher sind kommunitäre Organisationsformen realistisch, werden größere und qualitativ andere individuelle Freiräume möglich und desto irrelevanter wird auch die Form der Einkommensverteilung.

Sicherlich kann diese eher quantitative Betrachtung allein nicht zu hinreichenden Kriterien führen. Deutlich ist indes, daß sich die Qualität der einzelnen Ansätze in einem Bereich zwischen diesen beiden Extrempunkten erweisen muß. Hier muß beurteilt werden, welche Konzepte wie durchsetzbar und finanzierbar sind, was sich als illusionär erweist und welche Konzepte labiler sind als andere und ggf. deshalb trotz einzelner Vorteile abzulehnen sind. Auch die Vorschläge aus dem links-alternativen Umfeld bedienen sich in wesentlichen Momenten 
des gleichen Instrumentariums wie in neokonservativen Konzeptionen. Ganz offensichtlich kann dieses Vorgehen aufgrund der intentional konträren Zielperspektiven nur mit der Hoffnung begründet werden, daß es innerhalb der Bandbreite unterschiedlicher Leistungsniveaus markante Bereiche gibt, in denen Quantität in Qualität umschlägt. Wie obige Überlegungen gezeigt haben, ist diese Hoffnung tatsächlich berechtigt; allerdings ergibt sich aus der konzeptionellen Ähnlichkeit der Modelle an dieser Stelle folgende Schlußfolgerung: Die »Position 'jede Höhe des ME ist besser als keines' ist ... gefährlich « (Schreyer 1986, S. 161). Auf keinen Fall darf der Prozeß der Institutionalisierung eines anderen Transfermodells nach dem Muster von Tarifverhandlungen verlaufen ${ }^{12}$; zumindest die »Integrationsmodelle«(von Transfer- und Steuersystem) sind von der Richtung ihrer gesellschafts- und sozialpolitischen Wirkung her zu sehr von der Höhe der Transfers abhängig und allein deshalb zu labil, um den tatsächlichen materiellen Umfang der Leistung dem politischen »Tauziehen« überantworten zu können.

Allein aus dieser Überlegung heraus wird deutlich, daß aufgrund der zu befürchtenden Labilität eines anderen Transfersystems in der Realisierungsphase eine solche Neuadjustierung nur wünschenswert sein kann, wenn sie von den Umverteilungswirkungen her deutlich über dem Niveau des bestehenden Sozialsystems liegt. Solange dies nicht zu gewährleisten ist, besteht immer die Gefahr, daß sich eine reduzierte Variante mit umgekehrten Vorzeichen durchsetzt: Reformversuche müssen aus der Kritik des bestehenden Transfersystems beraus konzipiert werden. Aus dieser Einsicht und weil das Sozialsystem durch seine existenzsichernden Funktionen überaus empfindlich gegenüber kleinsten Erschütterungen reagiert, muß nach dem Prinzip verfahren werden, $»$ daß mit dem Einbau ... (einer, F.M.) Grundsicherung in das Sozialsystem keine Verschlechterungen an anderen Stellen des Systems auftreten dürfen (Bust-Bartels 1985 a, S. 172; vgl. auch Hanesch 1984, S. 127 f.).

Natürlich verhindert dieses Prinzip durchaus nicht, bestehende Privilegien abzubauen bzw. neue Personengruppen einzubeziehen, so daß sie in wesentlich größerem Umfang als bisher zur Umverteilung herangezogen würden (vgl. dazu die Vorschläge von Opielka [1985] zur Ausweitung der Sozialversicherungspflicht). Mit der Abkehr vom Sozialversicherungsgedanken und mit der Steuerfinanzierung der Mindestsicherung allein ist es noch nicht getan. Es ist die logische Konsequenz auf der Finanzierungsseite der ME-Forderung (ein weiteres quantitatives Kriterium mit qualitativen Folgen), die Notwendigkeit einer grundlegenden Reform des Steuersystems zu betonen (vgl. Adamy/Bäcker 1985; Bäcker 1985). Solange die gesellschaflichen Kräfteverhältnisse nicht den Einstieg in ein zumindest klassenneutrales Steuersystem (das wenigstens umverteilungspolitisch die gleiche Meßlatte anlegte) ${ }^{13}$ zulassen, erscheint auch ein Transfersystem, das neue qualitative Impulse setzt, wenig wahrscheinlich. Auf jeden Fall ist es schwer, die Auswirkungen der in der Regel mittelschichtorientierten Vorschläge zur Finanzierung der ME-Forderung abzuschätzen.

Wenn die Mindestsicherung alternativ zum heutigen Sozialsystem in einer einzigen Leistung aufgehen soll, gilt es bezüglich der Höhe der Transfers einer weiteren Gefahr zu begegnen: Selbst bei höherem Sicherungsniveau könnte (durch die Eingleisigkeit der Absicherung) im Zuge einer solchen technokratischen Effektivierung oberbalb der Basissicherung wieder Marktgesetzen in Bereichen zum Durchbruch verholfen werden, wo sie - trotz aller Zersetzung des Bedarfsprinzips in den letzten Jahren - durch den Sozialstaat ansatzweise schon zurückgedrängt worden waren. Durch die Integration des Steuersystems mit dem Transfersystem werden über ein Strukturmoment wie Automatismus der Leistungsgewährung individuell höchst unterschiedliche Lebenslagen auf den Indikator vermeintlicher materieller Vergleichbarkeit redu- 
ziert und dabei Bedarfsgesichtspunkte einer zentralen Sozialbürokratie überantwortet. Eine bei Bedarf nach qualitativen Gesichtspunkten durchzuführende Einzelfallbegutachtung, die auch besondere Notlagen würdigen könnte, würde so durch einen falsch verstandenen Gleichheitsgrundsatz starren finanziellen Imperativen geopfert (vgl. auch Schwab 1984, S. 82). Insgesamt bedeutet dies nichts anderes, als daß das Leistungsniveau in diesem Fall deut lich höher sein müßte, als bei mehreren hintereinandergeschalteten Sicherungssystemen, um insgesamt den gleichen Sicherheitsstandard garantieren zu können (vgl. auch Hanesch 1985, S. 80 f.). Bei einem Systemvergleich gilt es, diesen (Umrechnungs-)Faktor zu beachten. Der »sozialpolitische Rationalisierungseffekt « (Vobruba) der Integrationsmodelle muß schon deshalb äußerst kritisch eingeschätzt werden.

\section{Hoffnung auf unkonventionelle Lösungsansätze}

Das Zusammentreffen von »alternativen « Lebensentwürfen, »unkonventionellen« Lösungsansätzen und eher »marktorthodoxen« Vorstellungen kennzeichnet jene Gruppe von Vorschlägen, die über die Nutzung von Rationalisierungspotentialen im Sozialsystem Änderungen der Reproduktionslogik durch ein ME annähernd kostenneutral erzielen wollen. Scheinbar erfolgt hier schon vom Ansatz her eine Selbstbeschränkung auf die „Machbarkeit «, so daß die ME-Forderung mit Blick auf den anvisierten konservativen Bündnispartner weniger als Umverteilungsmaßnahme diskutiert wird; nur das Umverteilungsmodell soll völlig neu konzipiert werden, um bestimmte Effekte zu erreichen. Diese Suche nach »unkonventionellen« Lösungsansätzen, die einem »umfassenden historischen Konzept der Wirtschaftsreform « (Gretschmann 1985 a, S. 91), einer »ökologischen Sozialpolitik « (Gerhardt/Weber 1984, S. 43) oder auch der ersatzlosen Beseitigung der »für den Empfänger stigmatisierenden Bedürftigkeitsprüfungen« (Gretschmann 1985 a, S. 92) dienen sollen, bleibt den Idealen der Marktwirtschaft verhaftet: Die erhofften gesellschaftspolitischen Alternativen werden vorweiegend nach Effizienzkriterien abgeklopft, während der antizipierte Wandel anhand von KostenNutzen-Analysen argumentativ vorbereitet wird. Die Individuen werden vorrangig als Wirtschaftssubjekte begriffen, deren ökonomisches Handeln es im Sinne gemeinwohlrelevanter Nutzenmaximierung zu beeinflussen gilt ${ }^{14}$ : Individuelle Handlungskonzepte und »subsidäre Steuerungsformen « sollen die »Nutzung menschlicher Energien und materieller Ressourcen (Gretschmann 1985, S. 245) garantieren.

Dies kann nicht ohne Auswirkungen auf den Sozialstaat bleiben, so daß mit der Zeit öffentliche Dienstleistungen und Aufgaben in die private Sphäre rückverlagert werden können: der "voluntary non-profit«-Sektor übernimmt sozial-, versorgungs- und wohlfahrtspolitische Aufgaben (vgl. ebd.). Folgerichtig werden als sozialpolitische Zauberformeln die »neue Subsidiarität, kollektive Selbsthilfe (und, F.M.) gemeinschaftliche Selbstorganisation öffentlicher Aufgaben « (ebd., S. 244) entdeckt. In der Tendenz geht es also weniger um eine Ausweitung des Handlungsspielraums marginalisierter Bevölkerungsgruppen (durch deren materielle Existenzsicherung auf Basis einer gesellschaftlichen Umverteilungspolitik), als vielmehr um den Umbau des sozialen Sicherungssystems; "Leistungsalternativen und Finanzierungsalternativen sind gefragt « (ebd., S. 243).

Nicht ohne Grund stützen auch K.-U. Gerhardt und A. Weber (1984, S. 37 u. 50 ff.) ihre Option für eine NES auf deren Arbeitsanreiz-Effekte bei niedrigem Einkommen. Ja, sie stellen sogar ein Sinken der staatlichen Transferzahlungen in Aussicht, »In dem Maße, in dem Menschen selbstversorgend und sich gegenseitig helfend ihre Dinge wieder in die Hände nehmen, 
in dem Maße sinkt auch der Versorgungs-, Staats- und Sozialbedarf«(ebd., S. 43; Gerhardt 1985, S. 67): Die NES »ist günstiger als die herkömmlichen sozialpolitischen Regelungen ... Die kostenmäßigen Vorteile werden sich langfristig bemerkbar machen, indem der Staatsbedarf sinkt « (Gerhardt/Weber 1984, S. 56). Wie sehr die Autoren zur Erreichung öko-sozialer Ziele allein auf die strukturellen Effekte des ME setzen (also allein auf die Verteilungsform), zeigt ferner, wie mit der Krise des Beschäftigungssystems »libertär«(Gerhardt/Weber) umgegangen werden soll: Statt durch insgesamt unbefriedigende generelle Arbeitszeit verkürzungen, soll die notwendige Umverteilung der Arbeit mit Hilfe des ME durch individuelle Arbeitszeitverkürzungen (Teilzeitarbeit in "verschiedenen Formen « / "freiwillige Arbeitslosigkeit«) bewältigt werden (vgl. ebd., S. 22 ff.; Schwab 1984, S. 81 ff.; vgl. demgegenüber die umfassenden Überlegungen zur Arbeitszeitflexibilisierung bei Vobruba 1984, 1985 a, 1986). Auch hier geht es nicht um die materiellen Umverteilungswirkungen, sondern um deren strukturellen Effekte: die Optimierung der »Marktfähigkeit « der lebendigen Arbeit (s.o.) durch deren beliebige Teilbarkeit. Nicht die »Freiheiten« der Individuen, sondern die des Marktes sind das Ziel der Operation; mit der industriellen Reservearmee muß man nicht nur »leben«, sie ist sogar »für die Existenz des Systems der Lohnarbeit unabdingbar geworden «(Gerhardt/Weber 1984, S. 23; als wenn sie das nicht schon immer wäre). Schlußfolgerung der Autoren: Eben deshalb müssen neue Formen der materiellen Sicherung gefunden werden, um so »dem Kränkeln der Arbeitsgesellschaft" (ebd., S. 19) Rechnung zu tragen. Doch auch diese »monetäre Absicherung der Arbeitslosen« wird durch die Vorschläge dem notwendigen Umfang nach gefährdet, denn jene zeichnen sich genau durch die Labilität aus, wie sie oben beschrieben wurde. Tatsächlich scheint auch kein angemessenes Problembewußtsein für das Spannungsfeld zwischen Qualität und Quantität zu bestehen, denn alle emanzipativen Zielsetzungen werden in dem Vorschlag von Gretschmann (1985a, S. 93) konterkariert, wenn bei Wegfall eines großen Teils der Sozialtransfers ein Basiseinkommen von $500 \mathrm{DM}$ monatlich gefordert wird. Vor diesem Hintergrund bleibt folgende Perspektive unverständlich: »Gerade strukturelle (Dauer-)Arbeitslosigkeit scheint allein durch Minimumgarantieren bewältigt werden zu können. Dies deshalb, weil erst diese tatsächliche Wahlfreiheiten (!) - etwa zwischen Arbeit und Freizeit oder zwischen Lohnarbeit und selbstbestimmter Arbeit in alternativer Produktion - schaffen...« (ebd., S. 96).

All diese Momente führen final (nicht intentional) zu einer relativen Nähe zu neokonservativen Vorschlägen. Bei dem Grünen J. Wiemann (1985) können allerdings kaum noch Abgrenzungen vorgenommen werden. ME dient bei ihm der ngesellschaftlich gewollten generellen Subventionierung des Faktors Arbeit«(ebd., S. 42), was übersetzt nichts anderes bedeutet, als die Subventionierung der Lohnkosten der Unternehmen: "Jobsuchende können ihre Arbeitskraft zunächst billiger anbieten, das Mindesteinkommen bleibt ihnen ja erhalten... (ebd., S. 41). Durch diese Lohnsenkungen »vor allem in den unteren Bereichen«(ebd.) soll es vermehrt zu Neueinstellungen kommen, was in der Folge durch die "Struktur- und Niveauanpassung der Löhne an die Bedürfnisse der Unternehmen« (ebd., S. 42) das »Arbeitslosenproblem« lösen helfe.

Was hier als Variante der Neoklassik noch den Pluralismus der »grünen Bewegung « unter Beweis stellen mag, beweist sich bei J. Wiemann als bedenklich stimmende Naivität. Er erklärt nämlich die Sozialpolitik zum gesellschaftspolitischen Experimentierfeld, auf dem »unkonventionelle Lösungsansätze« in einem »trial-and-error-Prozeß auf ihre Leistungsfähigkeit«(!) (ebd.) getestet werden sollen. Sein Vorschlag ist der, man solle »das Mindesteinkommen in kleinen Raten über einen längeren Zeitraum gestreckt erhöhen und abwarten, wie die Menschen darauf reagieren...« (ebd., S. 39). 
G. Vrobuba kann man dieser Gruppe von Vorschlägen sicherlich nicbt zurechnen ${ }^{15}$; doch auch er teilt mit eher »marktorthodoxen « Ansätzen die Illusion, das ME könne durch Etablierung eines »reinen Arbeitsmarktes« zur »Waffengleichheit von Angebot und Nachfrage « führen (vgl. Vobruba 1986, S. 51): Entgegen der objektiv bestehenden Asymmetrie von Arbeit und Kapital auf dem Markt sollten auf Basis des ME die Bedingungen eines »marktmäßigen« Arbeitsangebots erst noch geschaffen werden. Doch auch einem solchen "pragmatischen Konzept zur Basissicherung «(Vobruba 1985), das beim Schielen auf »politische Bündnisgenossen « mit dem »reinen Arbeitsmarkt« spielt (vgl. auch Glotz 1986, S. 147) und das der Befürchtung, mit einer materiellen Existenzsicherung könne die Arbeitsbereitschaft zerstört werden, ein subtiles Arbeitsanreiz-System (»Leistung muß sich lohnen«, ebd., S. 59) gegenüberstellt, dürfte die »gesellschaftspolitische Grenzüberschreitung von epochaler Bedeutung» (ebd., S. 61) nur schwer gelingen. Tatsächlich ordnet die Absicht, mit einem ME das Arbeitsangebot in »berechenbarer Weise (ebd.) zu verringern, dieses einmal mehr den sachlichen Strukturnotwendigkeiten des kapitalistischen Arbeitsmarktes (auf dem Arbeit nur als abgeleitete Größe nachgefragt werden kann) in unbedingter Weise unter, statt umgekehrt den Versuch zu unternehmen, Freiräume der Person durch gezieltes sozialpolitisches Handeln gegen den kapitalistischen Arbeitsmarkt durchzusetzen. Ohne am tatsächlichen Bedarf der Individuen orientierte qualitative Prinzipien, die auch in der Praxis einer rein sachgesetzlichen Logik widerstehen, kann Sozialpolitik unmöglich auch nur den »Einstieg « in transformatorische Prozesse flankieren.

\section{Das Mindesteinkommen - systemadäquate Forderung mit systemsprengendem Charakter?}

An entsprechenden Prinzipien und Forderungen, die Freiheiten gegen die Kapitallogik behaupten bzw. in ihrer Summe gar das »Reich der Freiheit « (Marx) einleiten könnten, fehlt es in der ME-Debatte insgesamt sicherlich nicht; auch, daß die Höhe der garantierten Grundsicherung von entscheidender Bedeutung für ihre gesellschaftlichen Effekte ist, steht zumeist außer Frage.

Doch wie soll die Umsetzung konkret gestaltet werden? Wie und in welchem Zeithorizont sind die verschiedenen Forderungen durchsetzbar? - und schließlich: Wer setzt sie durch, wer ist der Adressat und was ist das Kernziel der ME-Forderung?

In der Vergangenheit ist die Diskussion vorwiegend entlang der ersten Frage geführt worden - also dem Wie der Ausgestaltung der Forderung und deren technischen Details. Aber auch schlüssige Antworten auf die anderen Fragen müssen im Rahmen einer Strategiediskussion erst noch gefunden werden.

So ist die Frage nach dem Adressaten der Forderung häufig sicherlich zu früh mit »dem Sozialstaat « beantwortet worden. Die damit antizipierte Sozialreform scheint zudem als zentrales Mittel der Transformation der Gesellschaft strategisch überschätzt zu werden. ${ }^{16} \mathrm{Z}$ war wird versichert, das garantierte ME sei kein "gesellschaftspolitisches Patentrezept «(Opielka/Vrobuba 1986a, S. 14), doch Inhalte und Zielsetzungen des jeweiligen Forderungskatalogs unterstützen diese Annahme. Egal ob die »ausbeutungsüberwindende Strategie (Opielka 1985) des ME betont wird, oder, daß es »revolutionär sei, doch keine Revolution erfordere (Opielka 1985a, S. 58), oder ob der staatlichen Politik, in der die GRÜNEN »Machtpositionen« übernehmen müßten, eine »Katalysatorwirkung (Opielka 1985c) zugesprochen wird - eine systematische Trennung von revolutionstheoretischen Überlegungen und sozialpolitischen Fragen wird bei M. Opielka an zentralen Stellen nicht vollzogen. Mit dem ME wird 
versucht, auf der Verteilungsebene nahezu die gleichen Wirkungen zu erzielen, wie sie eine Vergesellschaftung der Produktionsmittel haben könnte (vgl. auch C. Offe: »Abkopplung der Verteilungsfrage von der Produktionsfrage «). ${ }^{17}$ Bei M. Opielka gipfelt die Sozialstaatsillusion $^{18}$ in dem Ziel, die notwendige gesellschaftliche Arbeitsumverteilung über eine steuerfinanzierte Sozialdividende als »überbetrieblichen Lohnausgleich « zu realisieren: die Strategie der »20-Stunden-Normalerwerbswoche" - verbunden mit einem Grundeinkommen von $1000 \mathrm{DM}$ - soll in einem darüber hinaus ökologischen und geschlechtsspezifischen Forderungen gerecht werdenden »Recht auf eigene Produktivmittel« kulminieren. (Opielka 1985; ders. $/$ Stalb 1986) ${ }^{19}$

Es sind u.a. strategische Überlegungen, auf die sich die Kritik an diesen Vorschlägen stützt. Das ME wird als Mittel der Systemtransformation diskutiert, ohne entsprechend zu reflektieren, daß die Entfaltung der emanzipativen Wirkungen der materiellen Grundsicherung die Überwindung der Systemlogik in einigen Bereichen bereits zur Voraussetzung hat. Dies bedarf der Erläuterung: Das spezifische und die innere Logik der überaus weitreichenden Hoffnungen der Sozialutopie Opielkas besteht gerade darin, daß eine emanzipatorische Perspektive dem »Bürgergehalt» bzw. dem Konzept der »egalitären materiellen Grundsicherung« (Offe 1984, S. 355) nur abeinem bestimmten Niveau der Umverteilung (bzw. des garantierten Grundeinkommens) zugesprochen werden kann - darunter gehorcht es seinen konservativen Baumeistern. Folgerichtig wird der sozial-gestalterische Anspruch des Konzepts auch an die Verwirklichung der gesamten ME-Forderung gebunden und nur in diesem Kontext diskutiert. So entspricht auch dies der inneren Logik: Vor einer möglichen Entfaltung der »transformatorischen « Potenzen der durch den gesellschaftlichen Strukturwandel »überschüssigen « lebendigen Arbeit und vor den befreienden Wirkungen einer alternativen Reproduktionslogik - die zusammen mit der bestehenden Herrschaftslogik, oder sei es auch »nur « mit der »Verteilungsfragex brechen könnten - steht die entsprechende Gestaltung der materiellen Rahmendaten, d.h. die Durchsetzung einer hinreichenden garantierten Mindestsicherung. Doch gerade der dazu notwendige Prozeß wird von seinen qualitativen Bedingungen her nicht ausreichend reflektiert: In seiner Staatszentriertheit setzt ein Teil der vorliegenden Ansätze auf eine enorme Ausweitung der staatlichen Umverteilung und auf weitreichende Staatseingriffe (vgl. auch Opielka/Vrobuba 1986a, S. 14), was in diesem Ausmaß dem bestehenden Staatsapparat allerdings eine Unmöglichkeit aufbürden müßte. Die materielle Bedeutung einer staatlichen Sozialpolitik ist unbestritten, jedoch kann man die »Verteilungsfrage" nicht von der "Produktionsfrage « trennen, obne die "Systemfrage« zu stellen; man muß nicht die gesellschaftliche Gleichverteilung ${ }^{20}$ fordern, um an die Systemgrenzen zu stoßen! Trennt man einmal die revolutionstheoretische Essenz der Konzeption von ihren sozialpolitischen Implikaten, so zeigt sich die ausgeklammerte Durchsetzungsperspektive in folgender Aporie: Ohne Frage stellten die Wirkungen des Konzepts der Sozialdividende, wie es von M. Opielka vorgetragen wird, die Systemlogik nachhaltig in Frage, doch: wer ein ME in der Form und in der Höhe durchsetzen kann (wie es dazu notwendig ist), braucht es nicht mehr durchzusetzen! Insofern gilt die Schlußfolgerung von M. Greven (1986, S. 67), die dieser in einem umfassenderen Kontext gezogen hat, auch hier: »Nirgends ist eine Konzeption ersichtlich, die in haltliche Fragen mit Strategien des Machterwerbs konstruktiv verbindet. « Ergänzend kann man sagen, daß es zumindest klärungsbedürftig bleibt, was Formeln wie »nicht-reformistische Reformen« oder "radikales Reformprogramm " (Offe $)^{21}$ inhaltlich austragen sollen. Die obige Argumentation impliziert die hier vertretene These, daß die beiden Integrationsmodelle - werden sie mit emanzipativen Erwartungen verbunden - untauglich für eine 
schrittweise Einfübrung sind: Für den Fall einer nur teilweisen Durchsetzung der Forderungen (in (a) Ausgestaltung und (b) Höhe), müßte also die Anfälligkeit der beiden Konzepte der grundsätzlichen Neugestaltung bzw. Vereinheitlichung des Sozialsystems gegenüber einer konservativen Vereinnahmung im Durchsetzungsproze $\beta$ nachgewiesen werden; d.h. vereinfacht, daß gezeigt werden muß, welche Wirkungen reduzierte Varianten der Forderung hervorrufen können:

(a) Ganz offensichtlich sind die Weichen in Richtung »Zwei-Drittel-Gesellschaft « gestellt. Von zentraler Bedeutung zur Beurteilung eines jeden Reformvorschlags muß deshalb die Beantwortung der Frage sein, in welcher Form diesen Spaltungstendenzen entgegengewirkt werden kann.

Durch ein ME läßt sich zwar diese Spaltung mehr oder minder erträglich finanziell abfedern, nicht aber durch ein ME allein et wa verhindern: die gesellschaftlichen Polarisierungstendenzen resultieren auch aus substantiellen Änderungen der Produktionsstruktur. „Massenarbeitslosigkeit kann nicht per Mindesteinkommen zur 'Befreiung von falscher Arbeit' uminterpretiert werden.«(Bleicher 1984 S. 76) Im Gegenteil: Eine isolierte ME-Strategie dürfte die Spaltung sogar zementieren, weil sie dadurch physisch eher erduldbar wird. Eine isoliert am Recht auf Einkommen orientierte Strategie kann in der Tendenz nichts anderes als den Versuch bedeuten, der gesellschaftlichen Ausgrenzung eines wachsenden Teils der Bevölkerung durch Kompensation (z.B. Subsistenz auf Minimalniveau) der Symptome zu begegnen (logische Fortsetzung der Tradition der Armenpolitik). Je nach Umfang der materiellen Ausgestaltung eines solchen (Reparatur-)Konzepts führt dies zu völliger sozialer Deprivation oder - im et was »günstigeren « Fall - zur Festigung der »dualistischen Spaltung zwischen einem hochtechnisierten, -qualifizierten und -bezahlten primären Beschäftigungssektor und einem arbeitsintensiven, niedrig bezahlten, in großen Teilen auf selbständiger Basis organisierten sekundären Beschäftigungssektor (Familien- und Kollektivbetriebe)«(Hanesch 1984 S. 153 f.; vgl. auch S. 129 ff.).

Demgegenüber muß nach Lösungen gesucht werden, die jedem eine Perspektive auf das Beschäftigungssystem ermöglichen, denn nur so können die gesellschaftlichen Spaltungstendenzen abgemildert, kann der zunehmenden Differenzierung von Interessenlagen des heteronomen Teils der Gesellschaft durch eine gemeinsame Politik alter und neuer sozialer Bewegungen begegnet werden und nur so kann das grundlegende Recht auf »Teilhabe und Teilnahme an gesellschaftlicher Normalität« (vgl. P. Townsend 1979, zitiert nach Oplieka 1984) verwirklicht werden: "... in einer arbeitsteiligen Gesellschaft (ist; F.M.) die Möglichkeit, Erwerbsarbeit auszuführen, der unumgängliche Weg zur gesamtgesellschaftlichen Eingliederung und Gleichberechtigung " (Gorz 1985, S. 61). Solange gesellschaftlich das Recht auf Arbeit nicht verwirklicht ist, kann eine sozial verantwortliche Lösung - entgegen einer »Entkopplung von Arbeit und Einkommen « - nur darin bestehen, das Recht auf Einkommen als Lohnersatzleistung in der einen oder anderen Form an das Erwerbssystem anzubinden und die gesellschaftliche Arbeitsumverteilung durch weitere Arbeitszeitverkürzung zu betreiben!

Demgegenüber werden Einkommen, die obne systematische Verbindung zur Produktion gesellschaftlichen Reichtums erzielt werden, niemals frei sein vom Nimbus des "großzügigen Opfers « der Priviligierten; in der Tendenz führen sie zu Individualismus, zu Polarisierung, zu sozialer Stigmatisierung und sind zumeist zu niedrig für ein menschenwürdiges Leben (vgl. auch die Argumentation zum »Mißbrauchsvorwurf « bei Vobruba 1986, S. 41 f.). Dieser funktionale Zusammenhang wird zwar immer wieder mit Nachdruck betont; dennoch besteht die Gefahr der Aufspaltung in Konzeptionen der Integration von Steuer- und Transfersystem, 
die Umverteilungsmaßnahmen nicht substantiell mit einer drastischen Umverteilung der Arbeit verknüpfen. ${ }^{22}$ Solange das Recht auf Einkommen und das Recht auf Arbeit - wenn auch gleichberechtigt - nebeneinander gefordert und verschiedene Strategien zu ihrer Realisierung diskutiert werden, ist nicht zu gewährleisten, daß sie nicht auch als Alternativen verstanden werden, bzw. daß im Durchsetzungsprozeß nur die eine Forderung Bestand hat. Diese Gefahr besteht auch fort, wenn vorgeschlagen wird, das eine durch das andere zu erreichen: Erst die Entkopplung von Arbeit und Einkommen durch ein garantiertes ME im Subsistenzbereich und dann das Recht auf selbstbestimmte Tätigkeit durch die »Befreiung von falscher Arbeit« (vgl. z.B. Schmid 1984); oder: Durch ein »wohldosiertes« Basiseinkommen dessen "Arbeitsmarkt-Entlastungseffekte" (Vobruda) so regulieren, daß sich Arbeitsangebot und -nachfrage tendenziell ausgleichen. (vgl. Vobruda 1986, S. 46; Offe 1984, S. 356)

(b) Soll die Tauglichkeit eines Einkommenssicherungsmodells untersucht werden, muß man realistischerweise v.a. der Annahme nachgehen, daß bei einem bestimmten gesellschaftlichen Durchsetzungshintergrund das wohlüberlegte Niveau einer Grundversorgung (wie es heute gefordert wird) sich nicht bzw. nicht in einem Schritt wird durchsetzen lassen. Bei einer solchen nur teilweisen Durchsetzung der ME-Forderung ist die Richtung, in die die dem jeweiligen Konzept immanenten Instrumente des Arbeitsanreizes wirken, entscheidend für die Abschätzung zu erwartender Wirkungen.

Ein Großteil der mit der Sozialdividende und mit der NES verbundenen Hoffnungen stützt sich auf die Form der Transfervergabe: Durch die (abgesehen von der Höhe des verfügbaren Einkommens) kriterienlose Ver. bzw. Berechnung der Transfers, deren automatische Verteilung und durch die grundsätzliche, bruchlose Kombinierbarkeit von Transfer- und Erwerbseinkommen sollen die dem bestehenden Sozialsystem inhärenten Selektions- und Zwangsmechanismen zugunsten größerer individueller Freiräume umgangen werden. Bei einem garantierten Einkommen, das als bedarfsdeckendes Volleinkommen konzipiert wäre, könnte diese Zielsetzung mit Sicherheit erreicht werden; doch so optimistisch fallen selbst die umfassenderen MEKonzepte nicht aus.

Demgegenüber sind allerdings selbst bei einem Niveau, das deutlich über den konservativen Zielvorstellungen liegt, unerwünschte materielle Nebenwirkungen zu befürchten, die den Automatismus des Leistungsbezugs und die damit verbundenen emanzipativen Erwartungen unter den Bedingungen des kapitalistischen Arbeitsmarktes als nur schwer vereinbar erscheinen lassen: Beide Integrationsmodelle sind mittelfristig an der Sicherung eines (armutsverhindernden) Mindestbedarfs orientiert; darüber hinausgehende Bedürfnisse müssen im Medium der Erwerbsarbeit befriedigt werden. In beiden Konzepten wird der Übergang zwischen Erwerbsarbeit und Erwerbslosigkeit fließend gestaltet; die Höhe der Transfers wird bei der NES direkt (monatl. Festsetzung) und bei der Sozialdividende indirekt (Steuerberechnung) der Einkommenssituation angepaßt. Erwerbstätigkeit wird in der Weise »belohnt«, daß zusätzliches Arbeitseinkommen nicht vollständig ${ }^{23}$, sondern nur anteilig (NES) auf das Transfereinkommen angerechnet oder ihm sogar - vermindert um einen relativ hohen Steuersatz - hinzuaddiert wird (Sozialdividende).

In dem Maße aber, in dem die Grenzen zwischen Erwerbsarbeit und Erwerbslosigkeit aufgeweicht werden, besteht auf der Ebene des kapitalistischen Arbeitsmarktes auch die Möglichkeit, Transfereinkommen als integralen Bestandteil des Erwerbseinkommens in die jeweiligen Kalkulationen einzubeziehen. ${ }^{24}$ (Genau dies wird ja z.T. auch beabsichtigt.) Ob diese Kalkulation allerdings für die Beschäftigtenseite aufgeht, ist zweifelhaft. Für einen Teil der Bezieher von Sekundäreinkommen würde es individuell rational, die notwendige Befriedigung von 
Bedürfnissen, die über das Mindesteinkommen hinausgehen, mit sinkenden Löhnen zu erkaufen. »Wird für einen Job relativ wenig Zeit aufgewendet, so werden eher schlechte Arbeitsbedingungen akzeptiert; braucht das erzielte Einkommen nicht allein den Lebensunterhalt zu sichern, so wird eher eine schlechte Bezahlung hingenommen.« (Schreyer 1986, S. 160) Je niedriger das durchgesetzte Sicherungsniveau, desto mehr führt der immanente $Z$ wang zu ergänzender Erwerbsarbeit zu zusätzlichen Arbeitsanreizen sowie zu erhöhtem Druck auf die Löhne (vgl. auch Bust-Bartels 1985; Hanesch 1985).

G. Vobruba glaubt durch die Gestaltung von fließenden Übergängen zwischen Erwerbstätigkeit und Erwerbslosigkeit eine "Armutsfalle» zu vermeiden: "Die Armutsfalle entstünde dann, wenn das garantierte Grundeinkommen so organisiert wäre, daß bei geringfügigem bis mäßigem Arbeitseinkommen gleich das gesamte Transfereinkommen gestrichen wird. Das ist ein wesentlicher Konstruktionsfehler der derzeitigen Sozialhilfe. Dadurch entsteht für den einzelnen ein 'Sprungbereich', in dem sich die Arbeitsaufnahme absolut oder relativ nicht lohnt. ... Damit entsteht die irrationale Situation, daß die Arbeitsaufnahme trotz des Wunsches nach Arbeit und ( $\mathrm{Zu}$-) Verdienst unterbleibt. Dies ist die Armutsfalle.«(Vobruba 1986, S. 47) Was soll also »vermieden« werden: Armut oder die „Verweigerung der Arbeitsaufnahme«? Wohl eher letzteres: Durch die Kombinierbarkeit von Erwerbs- und Transfereinkommen lohnt sich die Arbeitsaufnahme "relativ« auch bei "geringfügigem bis mäßigem « Lohnniveau! Ob Armut mit einem ME verhindert werden kann, ist abhängig von den erzielten Verteilungspositionen und dem Sicherungsniveau, nicht aber von den materiellen Anreizen zur Arbeitsaufnahme.

Denselben »Konstruktionsfehler « der im Kern an Bedarfsgesichtspunkten orientierten Sozialhilfe erkannten auch Konservative und Liberale, als sie durch rationale Betrachtungen zu dem Schluß gelangten, daß im marginalen Beschäftigungsbereich die Löhne nicht unter eine bestimmte Grenze fallen dürfen, um noch zur Arbeitsaufnahme zu »motivieren«. Sie ermittelten eine »Wohlfahrtsmißwirtschaft « und analysierten ferner, daß die Sozialhilfe in der Regel alternativ zum Arbeitseinkommen gewährt wird, so daß die Löhne, wenn schon nicht deutlich, so doch zumindest spürbar über dieser Armutsgrenze liegen müssen, um noch »rational lohnend « zu sein. (vgl. Pfaff 1986; Gerhardt/Weber 1984; Friedman 1984) Aus diesen Gründen mußten zur Arbeitsaufnahme "motivierende « Sozialfürsorgemodelle konzipiert werden, damit sich "Leistung" auch in diesem Bereich »lohne" und damit der Preis der Arbeitskraft seine marktkonforme Flexibilität erhielte: Sozialdividende und negative Einkommensteuer.

Natürlich ist die Perspektive bei G. Vobruba und anderen Befürwortern der Integration von Steuer- und Transfersystem eine gänzlich andere! - Es sollten an dieser Stelle keine Grenzen verwischt werden; es sollte nur gezeigt werden, wie sehr sich die Argumentationsstrukturen ähneln und wie sehr im links-alternativen Strang der ME-Debatte von der Realisierung von Maximal-Zielen her argumentiert wird. Nur wer von optimalen Bedingungen ausgeht, kann wie M. Opielka hoffen, daß sich die Qualität der gesellschaftlichen Wirkungen der Sozialdividende »umkehrt « und daß sie »unter den Bedingungen von Lohnarbeit und Arbeitsmarkt (!) den Besitzlosen endlich eine wesentliche, ihre individuelle und kollektive Konfliktfähigkeit optimierende Option (eröffnet, F.M.): die uneingeschränkte Verweigerung des Angebots der 'Ware Arbeitskraft'« (1985, S. 145).

Festzuhalten bleibt also, daß die hier diskutierten Vorschläge zur Integration von Steuer- und Transfersystem aus dem links-alternativen Spektrum ihre Relevanz als Fernziele erst innerhalb eines umfassenden gesellschaftlichen Transformationskonzepts erlangen können. Sie 
sind systematisch auf lange Zeithorizonte ausgelegt und entwickeln ihre sozialutopischen Wirkungen nur, wenn sie von einer breiten gesellschaftlichen Bewegung in Höbe und Ausgestaltung entsprechend anspruchsvoll durchgesetzt werden können. Die Modelle der Sozialdividende und der NES werden leicht als Mittel der Transformation überschätzt; demgegenüber ist es aber unwahrscheinlich, daß sie Systemgrenzen schrittweise oder »aus sich selbst heraus überwinden können.

Es ist notwendig, in der aktuellen Debatte sehr genau zwischen der Diskussion sozialutopischer Entwürfe und den Auseinandersetzungen um realpolitische Strategien zu unterscheiden. Kurz- und mittelfristig muß jedenfalls nach anderen Lösungen als der grundlegenden Neukonstruktion des Transfersystems gesucht werden, wenn dennoch die zumeist stigmatisierende Praxis des Sozialstaats überwunden bzw. wenn sich den zweifellos emanzipatorischen Wirkungen einer restriktionslosen, allgemeinen Transferleistung auf ausreichendem Niveau perspektivisch angenähert werden soll. Festzuhalten bleibt auch, daß die diskutierten, auf Kombinierbarkeit von Erwerbs- und Transfereinkommen ausgelegten Vorschläge unter marktwirtschaftlichen Bedingungen des Verkaufs der Arbeitskraft die Gefahr in sich tragen, als willkommene Lohnsubvention funktionalisiert zu werden. Eben diese Gefahr birgt der schon erwähnte Vorschlag, ein garantiertes Grundeinkommen als »überbetrieblichen Lohnausgleich « für die Reduktion der Wochenarbeitszeit einzusetzen (Opielka 1985d; ders./Stalb 1986), um so die Forderung nach vollem Lohnausgleich fallen lassen zu können. Nicht minder problematisch präsentiert sich die Absicht, die Höbe des ME zum Instrument des Arbeitsanreizes zu machen, nämlich: den »Arbeitsmarkt-Entlastungseffekt« über die Transferhöhe zu »dosieren (Vobruba 1986, S. 46 f.) oder zu »feinregulieren (Offe 1984, S. 356). (vgl. dazu kritisch Glotz 1986, S. 146 f.; Schreyer 1986, S. 160)

\section{Die mittelfristige Perspektive}

Bei einer schrittweisen Einführung von NES oder Sozialdividende ließen sich die oben beschriebenen Gefahren nur umgehen, wenn jene Modelle anfangs zusätzlich, neben dem bestehenden Sozialsystem installiert würden, d.h. wenn das herkömmliche System so lang seine Aufgaben weiter erfüllte, bis eine Integration von Steuer- und Transfersystem (ohne nachteilige materielle Nebenwirkungen) den in sie gesetzten Ansprüchen gerecht werden kann (vgl. Opielka/Stalb 1986). Als weniger aufwendigere, weniger labile und deshalb letztlich auch realistischere Alternative dazu, ist der Vorschlag der Sockelung des bestehenden Sozialsystems zu verstehen.

Die »Sockelungsperspektive« setzt gerade nicht auf die Kombinierbarkeit verschiedener Einkommensarten und nicht auf die Neukonstruktion des gesamten Sicherungssystems, sondern versucht (aus der Kritik des bestehenden Systems heraus), den Ausbau der sozialen Sicherung gegenüber den Tendenzen zur Reduktion des Sozialstaates zu betreiben. Wesentliche Konstruktionsprinzipien sind die Formulierung von Ansprüchen auf das Erwerbssystem und die Betonung der Eigenständigkeit der sozialen Sicherung.

Die perspektivische Anbindung des Sozialsystems an die Produktion gesellschaftlichen Reichtums bedeutet allerdings nicht, wie M. Opielka und G. Vobruba einer "Sockelung" fälschlich ausschließlich unterstellen, daß »Transfereinkommen ... damit in Höhe und Dauer von der Position im Erwerbsleben abhängig ${ }_{\alpha}(1986 a$, S. 6) bleiben sollen. Dies kann mittelfristig nur für Einkommen deutlich über dem Niveau des ME gelten. Demgegenüber kann ein Recht auf Einkommen allerdings »eigenständig und unabhängig von der Erwerbsarbeit«(vgl. 
ebd.) unter den Bedingungen des kapitalistischen Arbeitsmarktes nur für das garantierte ME gelten; jenes darf wiederum nicht portionsweise dem Erwerbseinkommen hinzugerechnet werden können. Vielmehr muß der Sockelbetrag alternativ zum Erwerbseinkommen garantiert werden, d.h. es muß ein ausreichendes Mindest-/Sockeleinkommen für all diejenigen gewährt werden, die im Erwerbssektor keine bzw. keine ausreichende Beschäftigung finden. Entgegen der spezifischen, lohnarbeitszentrierten Selektivität bestehender Sozialsysteme müssen Sozialeinkommen in allen Lebenslagen eine "Teilhabe und Teilnahme an gesellschaftlicher Normalität « ermöglichen und eben deshalb schon allein ausreichend dimensioniert sein. Neben einer systematischen Anbindung an Strategien der Bewältigung der Beschäftigungskrise bietet darüber hinaus die Forderung, möglichst allen Arbeitslosen einen Anspruch auf als Lohnersatz konzipierte Sozialeinkommen einzuräumen, im Kontext konfligierender Interessenlagen weit eher die Gewähr für eine angemessene Höhe des Sockelbetrags, als eine allgemeine Grundsicherung (Sozialdividende). Diese muß zum einen immer für alle Gesellschaftsmitglieder (ohne Rücksicht auf den Grad der bereits bestehenden materiellen Absicherung) konzipiert und (vor-)finanziert werden, und ist zum anderen weit mehr der Interpretation unterworfen (vgl. die neokonservativen Vorschläge), als ein als Lohnersatz konzipiertes Transfereinkommen.

Als Reform des Warenkorbprinzips in der Sozialhilfe (SH) schlugen A. Hofmann und St. Leibfried schon 1980 vor, die Regelsatzformel direkt am gesellschaftlichen Durchschnittseinkommen zu orientieren: "So könnte beispielsweise der einfache Regelsatz 80\% des gesellschaftlichen Durchschnittseinkommens aller alleinstehenden Arbeiter/Angestellten betragen« (S. 275), statt "auf das durchschnittliche Netto-Arbeitsentgelt unterer Lohngruppen « (ebd.; vgl. auch $\ 22$ BSHG, "Deckelungsvorschrift «) bezogen zu werden; dies könne ein "Recht auf angemessene, selbst definierte Arbeit sstatuieren. Aber natürlich sind auch dies Maximalforderungen. Zwar gilt es zu bedenken, daß im Gegensatz zum enormen Umverteilungsvolumen einer Sozialdividende das Sockelungskonzept von einem deutlich geringeren Gesamtvolumen ausgeht und deshalb größere Spielräume für ein garantiertes Niveau der Transfers gewinnt, doch auch für dieses Konzept ist die Höhe der materiellen Mindestsicherung und damit der politische Durchsetzungshintergrund entscheidend.

Insbesondere dürfte folgende Überlegung zentraler Gegenstand von Auseinandersetzungen sein: Da die angestrebte Existenzsicherung in allen Lebensbereichen garantiert werden soll, »ist mit ihr eine Kopplung des Grundeinkommens an einen (faktischen) Arbeitszwang gleichgültig in welcher Form - nicht zu vereinbaren «(Hanesch/Klein 1986, S. 18). Das Konzept schließt auf der Ebene der Mindestsicherung ein »Recht auf Nichtarbeit« ein, wenn die Dominanz des Sozialversicherungsgedankens aufgehoben und wenn dem Bedarfsprinzip durch konsequente »Finalisierung der Sozialpolitik « ${ }^{25}$ endlich flächendeckend zur Geltung verholfen werden soll. Die Arbeitserzwingungsmechanismen in der Sozialgesetzgebung (v.a. die $\mathbb{\int} 25$ BSHG und $119 \mathrm{AFG}$ ) würden in ihrer kruden existenzgefährdenden Form entfallen. W. Wanesch und Th. Klein (1986) sprechen sich demgegenüber für "positive Anreize« durch einen »Verfügbarkeitsmehrbedarf«im AFG aus.

Damit zielt die »Sockelungsperspektive«z.T. auf dieselben Arbeitsmarkteffekte, wie ein Teil der Vorschläge zur Sozialdividende und zur NES auch - der existentielle Druck der industriellen Reservearmee soll entfallen, marginale Beschäftigungsformen sollen in der Tendenz aufgehoben werden. Ein entscheidender Unterschied besteht allerdings in den in der "Sockelung des Sozialsystems« angelegten Arbeitsangebotsreaktionen. Während bei den beiden Integrationsmodellen bei mangelnder Ausgestaltung alles auf Zuverdienst (mit all den Proble- 
men der Lohnsubvention) hinausläuft, kann die Sockelung in bestimmten Grenzen durchaus schrittweise eingeführt werden, ohne zu Nachteilen in anderen Bereichen zu führen. Da sie darüber hinaus nicht auf Kombinierbarkeit von Transfer-und Erwerbseinkommen ausgelegt ist, verspricht sie wegen der geringeren Gefahr der konservativen Vereinnahmung ${ }^{26}$ eher als die anderen Modelle, durch die weitere Relativierung der Arbeitspflicht materielle Spielräume zu eröffnen, die die Möglichkeiten der Subjekte vergrößerten, sich Rechenschaft über qualitative Fragen vor der Arbeitsaufnah me abzulegen: Die "Attraktivität des Arbeitsmarktes bzw. die Gestaltung gesellschaftlicher Arbeit "(Hofmann/Leibfried 1980, S. 275) könnte so in das Zentrum gesellschaftlicher Auseinandersetzungen geraten! ${ }^{27}$

Aus diesen Gründen wird kurz- und mittelfristig auch auf Sockelungskonzepte, die direkt an das heutige System anknüpfen, zurückgegriffen. (vgl. Hanesch/Klein 1986; Opielka 1985; ders./Stalb; Die GRÜNEN 1986; Arbeitsgruppe Sozialpolitisches Programm ... 1986) Minimalziel all dieser Vorschläge ist die Absicht, die Abhängigkeit der sozialen Sicherung von den vorausgehenden Bedingungen der Erwerbstätigkeit im unteren Einkommensbereich aufzuheben (partielle Entflechtung von "Arbeiten und Essen «) und das Leistungsniveau in diesem Bereich aufzustocken. Jeweils unterschiedlich definierte Mindestsicherungsniveaus sollen in die einzelnen Sozialversicherungszweige (insbesondere Arbeitslosen- und Rentenversicherung) integriert werden, Bedarfsermittlungen (i.d.R. durch Pauschalisierung) vereinfacht und die Unterhaltspflicht auf die Kernfamilie beschränkt werden. (Generationensubsidiarität nur noch bei minderjährigen bzw. in Ausbildung befindlichen Kindern; ansonsten nur noch Ehegattensubsidarität.)

Allerdings soll die angesprochene »Finalisierung« der sozialen Sicherung mit böchst unterschiedlicher Konsequenz betrieben werden. So sollen nach dem sozialpolitischen Programmentwurf der SPD (Arbeitsgruppe Sozialpolitisches Programm ... 1986) zwar die Kürzungen bei der Arbeitslosenunterstützung seit 1982 zurückgenommen werden und soll eine »umfassende soziale Grundsicherung installiert werden, doch bleibt der Entwurf insgesamt recht undifferenziert. Immer noch unklar bleibt, ob alle Lebenslagen materiell abgesichert werden sollen, oder ob es nicht doch nach wie vor um die Sanktion bestimmter idealtypischer Lebensläufe geht: Die in sich widersprüchliche Logik des AFG wird nicht angetastet und "die Leistungsbedingungen der heutigen Sozialhilfe werden bei der Grundsicherung in den Versicherungssystemen mit geringen Modifikationen übernommen; eine generelle Harmonisierung mit der Sozialhilfe ist nicht vorgesehen «(Hanesch/Klein 1986, S. 6). Zwischen den Zeilen scheint der Entwurf insgesamt eher von der Notwendigkeit der finanziellen Entlastung der Kommunen (SH) motiviert zu sein, als davon, konsequent Bedarfsgesichtspunkte unter stigmatisierenden Bedürftigkeitskriterien freizulegen. Sollte die Konkretisierung dieses Programmentwurfs diese Annahmen bestätigen, so würde er tatsächlich über eine »Ausweitung der Leistungsansprüche unter ansonsten unveränderten Bedingungen «(Opielka/Stalb 1986, S. 88) nicht hinauskommen und der Logik des Versicherungsprinzips verhaftet bleiben (vgl. Hanesch/Klein 1986, S. 6).

Das derzeit differenzierteste mittelfristige Konzept dürften W. Hanesch und Th. Klein in ihrem Gutachten für Die GRÜNEN (April 1986) vorgelegt haben (vgl. aber auch Opielka/ Stalb 1986, S. 87 ff.). Neben den schon angesprochenen Änderungen geht es in dem Vorschlag einer sintegrierten bedarfsorientierten Grundsicherung in AFG und BSHG im wesentlichen um folgendes:

- Es wird eine generelle Harmonisierung von BSHG und AFG angestrebt, indem über einen aktualisierten und strukturverbesserten Warenkorb (vgl. auch Transfer-Enquête-Kommis- 
sion 1981) eine Regelsatzanhebung umgesetzt werden soll, die zur Grundlage beider Systeme wird; erst durch einen einheit lichen Einkommensbegriff kann auch eine einheit liche Berechnungsgrundlage in beiden Systemen gewährleistet werden.

- Bei Wegfall der Möglichkeiten einer Aussetzung bzw. Streichung des ME, sollen alle Arbeitslosen nur an die Bedingung »Verfügbarkeit« geknüpfte, bedarfsbezogene Ansprüche erwerben, die um einen "Verfügbarkeitsmehrbedarf« über dem Mindestbedarf nach dem BSHG liegen; fehlt diese Bedingung, bleibt der Anspruch auf das ME im BSHG bei entschärfter Bedürftigkeitsprüfung bestehen. (Gerade auch die Bedingungen der Leistungsgewährung werden in dem Vorschlag reflektiert.)

- Die Versicherungsleistungen des AFG sollen (auf dem Niveau von 1982) bestehen bleiben; die im engeren Sinne funktionslos gewordene, bedarfsabhängige Arbeitslosenhilfe soll allerdings in eine bedarfsunabhängige Versicherungsleistung umgewandelt werden (entweder zeitlich unbefristet oder auf zwei Jahre begrenzt).

Insgesamt könnte auf diese Weise ${ }^{28}$ zumindest Armut bei Arbeitslosigkeit, aber idealiter auch in allen anderen Lebenslagen (die Funktionen des BSHG bleiben ja erhalten), wirksam vermieden werden - der Sicherungsschutz würde steuerfinanziert deutlich erweitert werden. (Zur Finanzierbarkeit vgl. Kap. 4 des Entwurfs von Hanesch und Klein.) Das Konzept enthält darüber hinaus Elemente, die als Grundlage für weitergehende Strukturreformen dienen könnten. Insbesondere eine bedarfsunabbängige, unbefristete ALH (die nach der vorgelegten Berechnung durchaus finanzierbar erscheint) würde für den überwiegenden Teil der Leistungsempfänger den "Lohnersatzcharakter «sozialer Leistungen sicherstellen. Schließlich ist das Konzept für den Kern seines Gestaltungsanspruchs an das Kriterium »Nicht-Erwerbstätigkeit« gebunden, was die oben beschriebenen, unerwünschten Arbeitsangebotsreaktionen vermeiden könnte.

Über die ME-Debatte ist es in den letzten Jahren gelungen, aus der sozialpolitischen Defensive wieder in die Offensive zu gelangen und den fiskalischen Sachzwangargumentationen eine fruchtbare Werte-Diskussion entgegenzusetzen. Die anfänglich teilweise euphorische Übernahme von im Kern markttheoretischen Argumentationsmustern gehorchenden Transfermodellen im links-alternativen Spektrum ist mittlerweile einer realistischeren Einschätzung des schmalen Grades möglichen emanzipatorischen Handelns gewichen - dennoch bleibt als letztlich ernüchterndes Fazit, daß entscheidende strategische Fragen noch immer völlig unbeantwortet sind:

Weder ist ein »historisches Subjekt « in Sicht, das die weitreichenden ME-Forderungen aufnehmen und durchsetzen könnte, noch besteht hinreichend Einigkeit darüber, wie der gegenwärtige Bestand an Forderungen überhaupt umgesetzt werden kann, noch ist geklärt, wie das Recht auf Einkommen mit dem Recht auf Arbeit perspektivisch »verkoppelt« werden kann. In welchem Umfang sollen Sozialleistungen in Zukunft völlig unabhängig von Erwerbsarbeit erzielt werden können? Wird die Ehegattensubsidiarität aufzuheben sein, und ist es wünschenswert, ein gänzlich von Anspruchsprüfungen unabhängiges Transfersystem zu installieren? Auch der zentrale Einwand gegen die "Sockelungsperspektive«, ob diese als »Reform der kleinen Schritte «nicht schließlich doch im Ansatz steckenbleiben muß, ist unbeantwortet: Tatsächlich muß auch hier die Forderung von M. Opielka und H. Stalb, daß »ein integriertes, in sich schlüssiges Reformkonzept ... auch für die 'ersten Schritte' unverzichtbar « (1986, S. 88) sei, auf ihre Verwirklichung hin untersucht werden.

Eine Strategiediskussion wird sich der Aufgabe stellen müssen, mittelfristige »Sockelungsansätze« sinnvoll mit länger- und langfristigen Perspektiven zu verschränken, und v.a. auch, die 
für den antizipierten Wandel notwendige Kontinuität sicherstellen zu müssen. Solange nicht sozialpolitische Imperative, die sich auch heute noch breiter Akzeptanz erfreuen, der Entwicklung von Kapitalverwertungsinteressen geopfert werden, wird die Zukunft eine Relativierung des lohnarbeitszentrierten, rigide kriterienbezogenen und realiter nicht angemessen bedarfsorientierten Systems der sozialen Sicherung erfordern. - Entscheidend wird sein, wer den sich auftuenden Gestaltungsspielraum mit Inhalten füllen kann.

\section{Anmerkungen}

1 Vgl. Friedman 1984; Rhys-Williams 1953; Otten 1977; Almsick 1981; Kausemann 1983.

2 Die großangelegten Versuche zur Einführung einer NES in den USA (vgl. dazu Almsick 1981; Gerhardt/Weber 1984), die allerdings aufgrund ihrer Modellannahmen nicht auf die bundesrepublikanische Situation übertragen werden können, waren überwiegend auf die Ermittlung der Änderung des Arbeitsmarktverhaltens von Fürsorgeempfängern ausgerichtet.

3 Diese Sichtweise ignoriert natürlich, daß Lohnnebenkosten integraler Bestandteil der »Arbeitskosten« (Wert der Ware Arbeitskraft) sind.

4 V.a. auf diesem Strang der Debatte soll das Schwergewicht der Auseinandersetzung weiter unten liegen.

5 Vgl. Opielka 1985, 1985b; Huber 1982; Gorz 1983; Gerhardt/Weber 1984; Gerhardt 1985; Schwab 1985.

6 Auf eine kurze Formel gebracht, scheint die Schlußfolgerung, die unter den gegebenen Umständen eine ökologische Entwicklung einleiten soll, etwa folgende zu sein: "Wessen menschen würdige Existenz unabhängig von Erwerbsarbeit gesichert ist, wird von selbst eher bereit sein, sich nützlichen Dingen und der Herstellung nicht-schädlicher Produkte zuzuwenden.«

7 Formelhaft:

- Lösung der »sozialen Frage« durch sozialpolitische Maßnahmen

- Transformation der Gesellschaft durch "Befreiung von der Lohnarbeit «

- Ökologischer Umbau der Gesellschaft

- Abschaffung der materiellen Voraussetzungen des Patriarchats aber auch:

— Erhöhung der »Marktfähigkeit « des Faktors Arbeit

- Ökonomische Effektivierung des Transfersystems

- Reduzierung der Staatsquote

8 Hier kann nicht auf die Geschichte der Forderung eingegangen werden; vgl. dazu im einzelnen Kausemann (1983), Almsick (1981), Bust-Bartels (1985), Gerhardt/Weber 1984 und Opielka (1985).

9 Für den finanziellen Umfang der Modelle, aber eben auch für die materielle Basis der antizipierten freiheitsstiftenden Momente ist es übrigens von entscheidender Bedeutung, auf welche Bezugssub. jekte die Maßnahmen zugeschnitten werden: Sind es die Hausbalte, fält ein Großteil möglicher Bezieher (zumeist Frauen) aus der Maßnahme heraus, während konsequenter Individualbezug (ohne weitergehende haushaltsbezogene Bedarfsprüfungen) zugleich die Aufhebung der Generationenund Ehegattensubsidiarität bedeuten und den Leistungsumfang gewaltig ausdehnen würde. (Nur um den Preis eines erheblichen Kontrollaufwandes sind Zwischenformen zu bewirken.)

10 Diese sozialutopischen Entwürfe nehmen eine ungemein wichtige Funktion ein, indem entgegen allen aktuellen Totalisierungstendenzen die grundsätzliche Gestaltbarkeit der sozialen Realität plastisch wird und Gestaltungsalternativen aufgezeigt werden. (vgl. Bust-Bartels 1985a)

11 Einzig einer Position könnte es rational erscheinen, ein anderes Transfersystem zu fordern, aber nichts an den gegenwärtigen Verteilungspositionen zu ändern: Folgt man der pointierten Analyse von P. Glotz in diesem Punkt, so geht es einem Teil der Diskutanten lediglich um die Alimentierung des informellen Sektors. Das Angebot an das System wäre dann in et wa folgendes: »... für 800 
(DM, F.M.) sind wir bereit, euch und eurem ganzen gräßlichen Arbeits- und Wirtschaftssystem den Rücken zu kehren und euch in Frieden zu lassen. (Glotz 1986, S. 143; vgl. auch Bust-Bartels 1985)

12 Bei den Diskussionen auf dem Programmkongreß zum »Umbauprogramm « der GRÜNEN am 26./27.4.86 in Hannover konnte man bei einer großen Anzahl der Teilnehmer bedenklich stimmende Erwartungen registrieren, die offensichtlich die grundlegende Reform des sozialen Sicherungssystems mit eben solchen Tarifverhandlungen verwechselten: "Man fordert einen bestimmten Betrag und wird im Laufe der Zeit halt' auf einen niedrigeren heruntergehandelt«. - Was diese Erwartung bei den Integrationsmodellen und der damit verbundenen Aufgabe umkämpfter Strukturen sozialer Sicherheit bedeutet, mag die Überlegung verdeutlichen, was passiert, wenn das ME nur »wenige» $100 \mathrm{DM}$ variiert ...

13 Aus Statistiken zur Einkommensverteilung gehen - eingedenk der methodischen Probleme der Quantifizierung und obwohl der Berichtszeitraum die sozial-liberale Sozialpolitik erfaßt (!) nicht nur "wachsende Diskrepanzen zwischen den Einkommen der sozialen Gruppen nach der Umverteilung (Costas 1985, S. 132) hervor, es zeigt sich auch die überproportionale Beteiligung der Arbeiter- und Angestelltenhaushalte an der Umverteilung: diese werden (1981) mit $43 \%$ herangezogen, während Beamte nur 25\%, Selbständige (im nicht-landwirtschaftlichen Bereich) nur 27\% ihrer Bruttoeinkommen zur Umverteilung beisteuern. (vgl. ebd., S. 131)

14 J. Schwab zielt auf die "Sicherstellung des Wirtschaftssubjekts als Marktteilnehmer durch ein BasisEinkommen« $(1984$, S. 84$)$ als sozialpolitische Perspekrive ab.

15 G. Vobruba $(1984,1985,1985$ a, 1986) setzt sich ganz eindeutig dadurch ab, daß er das Sozialsystem aus seiner Lohnarbeitszentriertheit lösen will und daß er die ME-Forderung nicht isoliert erhebt, sondern sie in ein umfassendes gesellschaftliches Gesamtkonzept integriert. Darüber hinaus erscheint das geforderte Leistungsniveau bei ihm ausreichend dimensioniert.

16 Vgl.v.a. Opielka 1984, 1985, 1985a, 1985b, 1985c, 1985d, Opielka/Stalb 1986; aber auch andere Beiträge in: Opielka/Vobruba 1986, sowie Berger 1984 und Offe 1984.

17 C. Offe in seinem Kurzreferat auf dem Programmkongreß zum »Umbauprogramm « der Grünen am 26.4.86 in Hannover.

18 Vgl. auch G. Grözinger (1986), der mit den verteilungspolitischen Instrumenten des Sozialstaates dazu anhebt, nahezu alle repressiven und funktionalen Elemente der kapitalistischen Vergesellschaftungsform »finanztechnisch zu neutralisieren«.

19 Neben dieser Staatszentriertheit bei der Durchsetzung von Transformationszielen zeigt sich die z.T. übertriebene Erwartungshaltung auch in dem Spannungsverhältnis zwischen dem eigentlichen Inhalt der Forderung und deren plakativen Formulierung, wie sie in den Anfängen der aktuellen Debatte verwendet wurde: Die Entkopplung von Arbeit und Einkommen. Tatsächlich unterstellt dieser "gedankenlose Satz « (Polster u.a. 1985, S. 34), daß die Kopplung von Arbeit und Einkommen ein wichtiges Organisationsmoment im Spätkapitalismus sei (vgl. Pfriem 1985, S. 73). Demgegenüber ist natürlich der Einwand von Polster u.a. zu beachten, „daß die bürgerliche Produktionsweise die individuelle Entkopplung von Arbeit und Einkommen gerade zur Existenzgrundlage hat" (1985, S. 34). Aus diesem Grund zielt emanzipatorische Politik ja auch gerade darauf, die gesellschaftlichen Zwangsgesetze aufzuheben, die durch das Auseinanderfallen von Zirkulations- und Produktionssphäre die Entkopplung von konkreter Arbeitsleistung und individuellem Einkommen in der Form des Arbeitslohns erst bewirken, (vgl. dazu aber Opielka 1985c, S. 63) Was gesamtgesellschaftlich gesehen hinter der Formulierung der "Entkopplung von Arbeit und Einkommen" stand, war natürlich die Hoffnung, die Sekundär-von der Primärverteilung im unteren Einkommensbereich abzukoppeln, bzw. möglichst viel Spielraum jenseits der Lohnarbeiterexistenz zu eröffnen - was die Formulierung allerdings assoziiert, ist die gesellschaftliche Entkopplung von Produktion und Distribution, also letzlich die Aufhebung des Lohnarbeiterverhältnisses.

G. Vrobuba (1986; vgl. im Gegensatz dazu 1984) ersetzt diese mißglückte Formel heute durch die treffendere Metapher der Entflechtung von Arbeiten und Essen, die der intendierten weiteren Relativierung der Arbeitspflicht weit mehr gerecht wird.

Vgl. dazu die Forderung von Blickhäuser/Molter 1986. 
Bei der Forderung nach einem garantierten Basiseinkommen ist v.a. auch der Verteilungsspielraum interessant: Nach M. Schreyer (1986, S. 165 f.) ergäbe die Gleichverteilung des Volkseinkommens von 1983 (Kinder mit dem Faktor 0,5 berücksichtigt) ein Pro-Kopf-Einkommen von 1896 DM monatlich; nach Abzug der Staatstätigkeit (-konsum) gar nur 1594 DM.

21 C. Offe in einem Kurzreferat, a.a.O.

22 In der aktuellen Debatte wird dieser Gefahr meiner Kenntnis nach nur in den beiden umfassenden sozialutopischen Entwürfen von A. Gorz (1983) und M. Opielka zu begegnen versucht.

Während es bei M. Opielka 1984 noch hieß: „Nur einem garantiertem Bürgergehalt ... läge die Idee zugrunde, daß Arbeit mit Einkommen nichts mehr zu tun haben darf« (S. 116), besteht er heute auf einer systematischen Verknüpfung der gesellschaftlichen Umverteilung von Einkommen »mit der Forderung nach einer Unverteilung der Arbeit « (1985c, S. 63). Die Gorzsche Perspektive eines garantierten Sozialeinkommens (s.o.) in einer sektorisierten Ökonomie versteht sich noch darüber hinaus durch die Einführung einer Jahres- und Lebensarbeitszeit als »logische Weiterentwicklung des gewerkschaftlichen Kampfes für Arbeitszeitverkürzung«(1985, S. 61): A. Gorz geht es darum, »ganz im Sinne der traditionellen Forderung der Arbeiterbewegung das Recht auf lebenslänglich gesichertes, normales Volleinkommen mit dem Recht auf die entsprechende Menge gesellschaftlich erforderlicher Arbeit unlösbar zu verknüpfen. Entkoppelt werden müssen also nich Einkommen und Arbeit, sondern Einkommen und Arbeitszeit. «(1985a, Teil II)

Diese Gesamtkonzeptionen sind zu unterscheiden von einer Vielzahl eher in sich geschlossener ME-Vorschläge, die sich zwar Wirkungen auf den Arbeitsmarkt versprechen, das Recht auf Arbeit aber letztlich als eigenständige zweite Forderung verstehen.

23 Zwar regelt der $\$ 115 \mathrm{AFG}$ schon heute bei Bezug von ALG und ALH zusätzliches Arbeitseinkommen zur Hälfte anzurechnen, doch führt dies aufgrund anderer Regelungen nicht zu einer Praxis, die etwa der NES vergleichbar wäre:

- Durch die Halbierung des zusätzlichen Einkommens nach Abzug von Steuern und Sozialversicherungsbeiträgen ergibt sich eine Quasi-Besteuerung von $70 \%$ und mehr;

- es wird sogar zu 100\% angerechnet, sobald das verfügbare Einkommen (Transfer-plus Arbeitseinkommen) $80 \%$ des früheren Nettoeinkommens übersteigt;

- dieses Einkommen muß noch dazu in weniger als 19 Stunden wöchentlich ( $\$ 102 \mathrm{AFG}$ ) erzielt werden, da sonst dem Begriff nach keine Arbeitslosigkeit vorliegt.

Diese Ausführungsbestimmungen führen in der Praxis dazu, daß formelle Erwerbsarbeit bei Arbeitslosigkeit nur marginal in Erscheinung tritt.

24 Dies gilt natürlich für beide Seiten des Arbeitsmarktes, nicht nur für das Angebot und dessen »Wahlmöglichkeiten zwischen Arbeit und Freizeit «!

25 Sozialleistungen sollen »nicht aufgrund vorhergegangener Ursachen - also: kausal - sondern gemäß definierter Zwecke erbracht werden « (Vobruba 1986, S. 45).

26 W. Hanesch und Th. Klein schlagen zwar auch Anreize zur Erwerbsarbeit vor (Teilanrechnung von $80 \%$ der Erwerbseinkommen und der Lohnersatzleistungen/Verfügbarkeitsmehrbedarf von 20 bzw. 30\% des Regelsatzes des Mindesteinkommens), um Ärbeitsbereitschaft grundsätzlich zu honorieren und um im Gegenzug Negativ-Sanktionen fallenlassen zu können, doch wegen der relativ hohen Anrechnungsrate von $80 \%$ ist eine kapitallogische Funktionalisierung des ME als Lohnsubvention kaum zu befürchten.

27 A. Bust-Bartels verspricht sich durch diese Maßnahmen sogar einen »Rationalisierungsdruck für die Unternehmen, der mehr bewirken würde, als alle bisherigen Programme zur Humanisierung der Arbeit « (1985a, S. 177).

28 Das Konzept müßte allerdings noch auf die anderen Sozialversicherungszweige (zur Mindesrente vgl. auch die GRÜNEN ... 1985) und auf das Steuersystem abgestimmt werden. - Der Arbeitskreis "Sozialpolitik « bei den GRÜNEN im Bundestag stützte sich bei der Vorstellung seiner Vorschläge im April $1986 \mathrm{im}$ wesentlichen auf das Gutachten von W. Hanesch und Th. Klein und modifizierte es nur unwesentlich; die Struktur des Konzepts blieb erhalten, so daß auf die Änderungen an dieser Stelle nicht eingegangen werden braucht. (vgl. Arbeitskreis "Sozialpolitik« 1986) 


\section{Literatur:}

Adamy, W./Bäcker, G. (1985): Der Maschinenbeitrag - ein Allbeilmittel für Rentenfinanzen und Arbeitsmarkt?, in: WSI-Mitteilungen 1/1985, S. 24-32

Adamy, W./Steffen, J. (1984): Arbeitslos gleich arm, in: WSI-Mitteilungen 10/1984, S. 574-581

Adler-Karlsson, G. (1979): Gedanken zur Vollbeschäftigung, in: MittAB 4/1979, S. 481-505

Albrecht, E. (1983): Unser soziales System ist teuer und unwirtschaftlich, in: Frankfurter Rundschau v. 27. August 1983.

Almsick, J.v. (1981): Die negative Einkommensteuer, Berlin

Arbeitsgruppe Sozialpolitisches Programm und Kommission Sozialpolitik beim SPD-Parteivorstand (1986): Die Zukunft sozial gestalten (Entwurf), Bonn

Arbeitskreis »Sozialpolitik « bei den Grünen im Bundestag (1986): Grünes Modell einer bedarfsorientierten Grundsicberung in allen Lebenslagen, Bonn (April) 1986 (vv Manuskript)

Bäcker, G. (1985): Mindestsicherung und Maschinensteuer: Wege zur Lösung der sozialen und finanziellen Defizite der lobnbezogenen Sozialversichering?, in: Soziale Sicherheit, 2/1985, S. 33-45

Baethge, M./Overbeck, H. (1986): Zukunft der Angestellten, Frankfurt/M.

Balsen, W./Nakielski, H./Rössel, K./Winkel, R. (1984): Die neue Armut, Köln

Barthes, R. (1982): Mythen des Alltags, Frankfurt/M.

Berger, J. (1984): Alternativen zum Arbeitsmarkt, in: MittAB 1/1984, S. 63-72

Biedenkopf, K. (1984): Die Erneuerung der sozialen Marktwirtscbaft, in: Kerber, W.: Arbeitswelt im Umbruch, Düsseldorf, S. 98-127

Bielke, I. (1978): Die sozialreformerischen Ideen von Josef Popper-Lynkeus, Tübingen

Bleicher, F. (1984): Erlesene Befreiungen. Mindesteinkommen als All-round-Lösung?, in: Kommune 12/1984, S. 75-77

Blickhäuser, A./Molter, M. (1986): Garantierte Lebenssicberung für Frauen, in: Opielka/Vobruba (1986 b) Bust-Bartels, A. (1984): Recht auf Einkommen?, in: Aus Politik und Zeitgeschichte 28/1984, S. 39-54 ders. (1985): Das Recht auf Einkommen - eine systemsprengende Reform?, in: Widersprüche, Heft 14, 1985, S. 25-54

ders. (1985 a): Das garantierte Grundeinkommen als Herausforderung der Sozialwissenschaften, in: Leibfried/Müller (1985)

Costas, I. (1985): Grundlagen der Wirtschafts- und Sozialstatistik, Frankfurt/M.

Dahrendorf, R. (1983): Die Chancen der Krise. Über die Zukunft des Liberalismus, Stuttgart

Die Grünen (1986): Umbau der Industriegesellschaft. Programm zur Überwindung von Erwerbslosigkeit, Armut und Umweltzerstörung (Entwurf), Bonn

Die Grünen/Seniorenschutzbund „Graue Panther (Hg.) (1985): Grundrente statt Altersarmut, Berlin/ Essen

Dießenbacher, H. (1985): Zur »Kolonialisierung « fremder Lebenswelten, in: Widersprüche, Heft 14, 1985, S. 9-24

Engels, W./Mitschke, J./Starkloff, B. (1974): Staatsbürgersteuer, Wiesbaden

Friedman, M. (1984): Kapitalismus und Freibeit, Stuttgart

Gerhardt, K.-U. (1985): Eigeninitiative und Sozialpolitik, in: Widersprüche, Heft 14, 1985, S. 61-70

ders./Weber, A. (1984): Garantiertes Mindesteinkommen. Für einen libertären Umgang mit der Krise, in: Schmid (1984), S. 18-67

Glotz, P. (1986): Freiwillige Arbeitslosigkeit?, in: Opielka/Vobruba (1986 b), S. 135-148

Gorz, A. (1980): Abschied vom Proletariat, Frankfurt/M.

ders. (1983): Wege ins Paradies, Berlin

ders. (1985): Abkopplung von Einkommen und Arbeit oder von Einkommen und A rbeitszeit?, in Kommune $5 / 1985$, S. $60-62$

ders. (1985 a): Über die Phantasie und das Ziel einer humanen Gesellschaft, Teil I u. II, in: Frankfurter Rundschau v. 23. u. 25. November 1985

ders. (1986): Garantierte Grundversorgung aus rechter und linker Sicht, in: Opielka/Vobruba(1986 b), S. 53-62 
Gretschmann, K. (1985): Sozialpolitik unter Veränderungsdruck, in: Opielka (1985 b), S. 243-252

ders. (1985 a): Garantiertes Minimum. Vom Recht auf Arbeit zum Recht auf Einkommen, in: Brun, R.

(Hg.): Erwerb und Eigenarbeit, Frankfurt/M. 1985, S. 85-99

Greven, M.Th. (1986): Von der Sozialleistung zum Bürgergebalt. Die Entprivatisierung des gesellschaftli. chen Reichtums, in: Opielka/Vobruba (1986 b), S. 63-69

Grözinger, G. (1986): Finanzierungsaspekte eines garantierten Grundeinkommens, in: Opielka/Vobruba (1986 b), S. $169-179$

Hanesch, W. (1984): Einkommenssicherung in der Krise, in: Schmid (1984), S. 121-142

ders. (1985): Anmerkungen zur Forderung nach einem Garantierten Mindesteinkommen, in: Widersprüche, Heft 14, 1985, S. 77-84

ders./Klein, Th. (1986): Eine integrierte bedarfsorientierte Grundsicherung in AFG und BSHG, Bonn (April) 1986 (vv Manuskript)

Hauser, R./Cremer-Schäfer, H./Nouvertné, U. (1981): Armwt, Niedrigeinkommen und Unterversorgung in der Bundesrepublik Deutschland, Frankfurt/M.

Hofmann, A./Leibfried, St. (1980): Historische Regelmäßigkeiten der Regelsätze-100 Jabre Tradition des Deutschen Vereins?, in: Neue Praxis 3/1980, S. 253-285

Huber, J. (1982): Die verlorene Unschuld der Ökologie, Frankfurt/M.

Kausemann, E.-P. (1983): Möglichkeiten einer Integration von Steuer-und Transfersystem, Frankfurt/M.

Kern, H./Schumann, M. (1985): Das Ende der Arbeitsteilung? - - Rationalisierung in der industriellen Produktion, München

Kollektiv Charles Fourier (1986): Das allgemeine Grundeinkommen, in: Opielka/Vobruba (1986 b), S. 235-242

Kommission »Zukunftsperspektiven gesellschaftlicher Entwicklung« (1983): Bericht, Stuttgart

Kronberger Kreis (1984): Mebr Mut zum Markt - Wege zur Erneuerung der Sozialen Marktwirtschaft, in:

Voss, R./Walter, N.v. (Hg.): Wohin treibt die Wirtschaft, Stuttgart 1984

Leibfried, St. (1986): Bedarfsprinzip und Krise sozialer Grundsicherung, in: Opielka/Vobruba (1986 b), S. $149-157$

ders./Tennstedt, F. (Hg.) (1985): Politik der Armut und Die Spaltung des Sozialstaats, Frankfurt/M. dies. (1985 a): Die Spaltung des Sozialstaats und Die Politik der Armut, in: dies. (1985), S. 13-37

Leibfried, St./Müller, R. (Hg.) (1985): Sozialpolitische Bilanz. Referate auf der Tagung der Sektion Sozialpolitik der Deutschen Gesellschaft für Soziologie am 3.-4. Mai 1985, Bremen 1985

Marson, P. (1985): Garantiertes Minimum und Weltmarkt, in: Brun, R. (Hg.): Erwerb und Eigenarbeit, Frankfurt/M., S. 100-107

Miegel, M. (1981): Sicherbeit im Alter, Stuttgart

Molitor, B. (1973): Negative Einkommensteuer als sozialpolitisches Instrument, in: Jabrbuch der Sozialwissenschaft, Bd. 241973

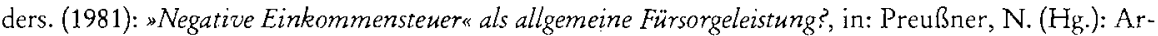
mut und Sozialstaat, München 1981.

Offe, C. (1984): Perspektiven auf die Zukunft des Arbeitsmarktes. "Orthodoxie", „Realismus" und "dritte Weger, in: ders.: "Arbeitsgesellschaft«: Strukturprobleme und Zukunftsperspektiven, Frankfurt/M. 1984 , S. 340-358

Opielka, M. (1984): Das garantierte Mindesteinkommen - ein sozialstaatliches Paradoxon?, in: Schmid (1984), S. 99-120

ders. (1985): Sozialreform obne Wirtschaftswachstum, in: Leibfried/Müller (1985), S. 127-161

ders. (1985 a): Jenseits von Armut und Kapitalismus, in: Widersprüche, Heft 14, 1985, S. 55-60

ders. (Hg.) (1985 b): Die ökosoziale Frage. Entwürfe zum Sozialstaat, Frankfurt/M.

ders. $(1985$ c): Umverteilung von Arbeit und Einkommen, in: Kommune 5/1985, S. 63-66

ders. (1985 d): Sozialpolitische Alternativen und die Zukunft sozialer Berufe, in: Schön, B. (Hg.): Die Zukunft der sozialen Berufe, Frankfurt/M. 1985

ders./Stalb, H. (1986): Das garantierte Grundeinkommen ist unabdingbar, aber es genügt nicht, in: Opielka/Vobruba (1986 b), S. 73-97 
Opielka, M./Vobruba, G. (1986 a): Warum das Grundeinkommen diskutieren?, in: dies. (1986 b), S. 5-15 dies. (1986 b) (Hg.): Das garantierte Grundeinkommen. Entwoicklung und Perspektiven einer Forderung, Frankfurt/M.

Otten, A. (1977): Die negative Einkommensteuer als sozialpolitische Alternative, Frankfurt/M.

Pabst, S. (1983): Für eine liberale Erneuerung der Sozialsysteme, in: Liberal, November 1983, S. 816-822

Pfaff, M. (1986): Garantiertes Einkommen oder garantierte Arbeit?, in: Opielka/Vobruba (1986 b), S. 28-35

Polster, W./Rieckmann, P./Voy, K. (1985): Lohnarbeit 2000. Über Segens- und Scbreckensvisionen der neuen Techniken, in: Kommune 3/1985, S. 27-34

Rehn, G. (1973): Die Gesellschaft der freien Wabl, in Külp, B./Stützel, W. (Hg.): Beiträge zu einer Theorie der Sozialpolitik, Berlin 1973

Rhys-Williams, J. (1953): Something to Look Forward to, London 1942, Teilabdruck in: dies.: Taxation and Incentive, London 1953

Roberts, K. (1986): Ein Plan zur Einfübrung eines Basiseinkommens, in: Opielka/Vobruba (1986 b), S. $98-105$

Schmid, Th. (1984) (Hg.): Befreiung von falscher Arbeit, Berlin

ders. (1985) (Hg.): Das Ende der starren Zeit, Berlin

Schreyer, M. (1986): Mindesteinkommen - Stolper-oder Meilenstein für eine grüne Zukunft?, in: Opielka/Vobruba (1986 b), S. 158-168

Schwab, J. (1984): Mindesteinkommen als sozialpolitische Perspektive, in: Schmid (1984), S. 76-85

Transfer-Enquête-Kommission (1981): Das Transfersystem in der Bundesrepublik Deutschland, Stuttgart Vobruba, G. (1983): Politik mit dem Woblfabrtsstaat, Frankfurt/M.

ders. (1984): Die Entkopplung von Arbeit und Einkommen, in: Widersprüche, Heft 12, 1984, S. 79-88

ders. (1985): Arbeiten und Essen. Die Logik im Wandel des Verbältnisses von gesellschaftlicher Arbeit und existentieller Sicherung im Kapitalismus, in: Leibfried/Tennstedt (1985), S. 41-63

ders. (1985 a): Wege aus der Flexibilisierungsfalle. Plädoyer für die Verbindung von Arbeitszeitverkürzung, Flexibilisierung und garantiertem Einkommen, in: Schmid (1985), S. 25-39

ders. (1986): Die Entflechtung von Arbeiten und Essen, in: Opielka/Vobruba (1986 b), S. 39-52

Wiemann, J. (1985): Einfach, sozial und ökologisch. Mindesteinkommen als ökonomischer Hebel grüner Po. litik, in: Kommune 3/1985, S. 35-42

Wiesenthal, H. (1985): Themenraub und falsche Allgeneinheiten, in: Schmid (1985), S. 9-24 